Notre Dame Journal of Formal Logic

Volume 35, Number 3, Summer 1994

\title{
Algebraic Study of Two Deductive Systems of Relevance Logic
}

\author{
JOSEP MARIA FONT and GONZALO RODRÍGUEZ
}

\begin{abstract}
In this paper two deductive systems (i.e., two consequence relations) associated with relevance logic are studied from an algebraic point of view. One is defined by the familiar, Hilbert-style, formalization of $\mathbf{R}$; the other one is a weak version of it, called $\mathbf{W R}$, which appears as the semantic entailment of the Meyer-Routley-Fine semantics, and which has already been suggested by Wójcicki for other reasons. This weaker consequence is first defined indirectly, using $\mathbf{R}$, but we prove that the first one turns out to be an axiomatic extension of WR. Moreover we provide WR with a natural Gentzen calculus (of a classical kind). It is proved that both deductive systems have the same associated class of algebras but different classes of models on these algebras. The notion of model used here is an abstract logic, that is, a closure operator on an abstract algebra; the abstract logics obtained in the case of WR are also the models, in a natural sense, of the given Gentzen calculus.
\end{abstract}

1 Introduction This paper intends to be a contribution to the study of the well known system $\mathbf{R}$ of Relevance Logic, and of a weaker companion system called here WR, from a precise point of view of algebraic nature. We will follow some wellestablished methods, such as those systematized in Blok and Pigozzi [5], Czelakowski [9], Wójcicki [26], 27], as well as newer methods, developed recently in full generality in Font and Jansana [16, but already in use for several years for many particular cases (see Bloom and Brown [6] and Font and Verdú [18]-[21], and also Font [15] for a survey). These not-so-well-known methods use abstract logics, that is, pairs $\mathbb{L}=\langle\mathfrak{A}, \mathrm{C}\rangle$ where $\mathrm{C}$ is a closure operator on the universe of the algebra $\mathfrak{A}$, as a kind of models of logics; they were introduced in Brown and Suszko $[7$ as a generalization of Tarski's early use of consequence operations on the formula algebra and also of the general theory of logical matrices (although already in 1962 Smiley 25 pointed out the need for them). The objects of study in this area of Algebraic Logic are called, somehow conventionally, deductive systems; by definition a deductive system is a 
finitary and structural closure operator on the algebra of formulas. Thus when we want to study a given logical system with these methods we have to turn it into a deductive system by some procedure, which might not be in accordance with the original - extra logical - motivations for introducing the logical system. This is what happens in the case of relevance logic: by definition a deductive system satisfies the Weakening Rule, which allows one to add hypotheses to a given derivation without invalidating it. This property is rejected by many scholars on the grounds that it precisely destroys relevance; these ideas end up with formalizations in the Gentzen style lacking some structural rule(s), and all our algebraic apparatus cannot be applied to them. In this paper we shall not be concerned with such points; the reader will find interesting readings on these and related issues in Došen and Schroeder-Heister [13].

The issue of formalizing the notion of relevant implication or entailment is surely not trivial. One of the predominant approaches in the literature has been to find a class of formulas concerning it (i.e., containing some formal connective of implication) which can be shown to represent faithfully all logically true statements about entailment. This can be done either semantically (by taking all formulas true in a suitable class of models) or syntactically (by taking all formulas provable in a given formal system). The famous system $\mathbf{R}$ was originally introduced (see Anderson and Belnap [1]) as the set of theorems of a Hilbert-style formal system. Then, by allowing inferences from hypotheses, the same formal system defines a deductive system, which is sometimes called official deducibility (cf. for instance [1], § 22.2.1 or Anderson, Belnap and Dunn [2], p. 169). This deductive system has already been studied from an algebraic point of view on p. 48ff. of [5] and Font and Rodríguez [17]; it is algebraizable, in the precise sense of the term introduced in [5], and the corresponding class of algebras has been identified in [17], where they are called relevant algebras or $\mathbf{R}$-algebras (they turn out to form an intermediate class between De Morgan monoids and De Morgan semigroups, two classes of algebraic models usually considered).

However, this is not the only possible approach to the problem of finding a deductive system faithfully representing the informal notion of entailment that system $\mathbf{R}$ tries to formalize. Actually, Wójcicki in [26, pp. 52-73 and [27], pp. 163-170 has made some insightful observations on the issue of how to define, in general, a deductive system from the set of theorems of some logical system. He has advanced a proposal along the following line: If we are to represent the (informal) notion of entailment by the (formal) notion of deduction, that is, by the metalogical connective " $\vdash$ ", then we can adopt as a definition of such a deductive system the idea that

The formulas $\varphi_{1}, \ldots, \varphi_{n} \in F m$ entail the formula $\varphi \in F m$

if and only if

the formula $\left(\varphi_{1} \wedge \ldots \wedge \varphi_{n}\right) \rightarrow \varphi$ is a theorem of $\mathbf{R}$.

Since this determines the consequences of only nonempty and finite sets of formulas, Wójcicki adds in 26 the conditions that the logic must be finitary and have no theorems (this last condition is replaced in [27] by the equivalent one that no formula is a consequence of each of the nonempty theories of the logic).

The relation of consequence just described is implicitly present in the works on the relational semantics devised by Routley, Meyer, Fine, and others. Indeed, these authors have proved a completeness theorem for $\mathbf{R}$ (see [2], $\S \S 48,51$ for details) that 
as a consequence yields that a formula of the form $\left(\varphi_{1} \wedge \ldots \wedge \varphi_{n}\right) \rightarrow \varphi$ is a theorem of $\mathbf{R}$ if and only if the formulas $\varphi_{1}, \ldots, \varphi_{n}$ semantically entail the formula $\varphi$, that is, if and only if $\varphi$ is satisfied, for some valuation, at any point in a model structure where all the $\varphi_{i}$ are satisfied. The reader may notice that the semantic entailment relation underlying this result coincides with Wójcicki's proposal.

Our main concern in this paper will be the algebraic study of the deductive system, which we call $\mathbf{W R}$, that arises in this way. Since $\mathbf{W R}$ and $\mathbf{R}$ are closely connected, this study will also furnish a context in which some further contributions to the algebraic study of $\mathbf{R}$ will be made.

Section 2 contains some general definitions and results, and in particular those concerning $\mathbf{R}$ and its algebraization, mainly taken from [17]. The class of $\mathbf{R}$-algebras is introduced, and its relationship with the deductive system $\mathbf{R}$ is described, allowing us to determine all the $\mathbf{R}$-filters on an $\mathbf{R}$-algebra. The general concepts and techniques of Universal Algebra and Lattice Theory can be found in Balbes and Dwinger 3] and Burris and Sankappanavar [8], and those of the Theory of Abstract Logics in Brown and Suszko [7].

Section 3 begins with the formal definition of $\mathbf{W R}$ and the proof that actually $\mathbf{R}$ is the axiomatic extension of WR having the Identity Law $p \rightarrow p$ as an additional axiom schema. Then a Completeness Theorem for WR is found, with respect to the class of matrices obtained from all $\mathbf{R}$-algebras and all their lattice filters plus the empty set (a similar one for $\mathbf{R}$ was obtained in [17] by taking just those lattice filters containing all elements of the form $a \rightarrow a$ ). We show that, unlike in the case of $\mathbf{R}$, we cannot put the larger class of De Morgan semigroups in the place of the class of $\mathbf{R}$-algebras in this Completeness result. Using it we prove that the $\mathbf{W R}$-filters on an $\mathbf{R}$-algebra are exactly all its lattice filters plus the empty set. We then show that WR is not algebraizable in the sense of [5], nor even protoalgebraic in the sense of Blok and Pigozzi [4]. This section ends with the determination of all the reduced matrices for $\mathbf{W R}$ and the proof that their algebraic reducts form exactly the class of all $\mathbf{R}$-algebras. Thus both deductive systems share the same associated class of algebras.

Section 4 is the central one. It begins with the definition of a Gentzen calculus $\mathcal{G}_{\mathbf{W R}}$ and of the deductive system $\mathcal{S}_{\mathbf{W R}}$ associated with it in a standard form. In order to prove the main result that $\boldsymbol{S}_{\mathbf{W R}}$ is exactly $\mathbf{W R}$ (thus obtaining an intrinsic formalization of $\mathbf{W R}$ ) we introduce and study the class of abstract logics which are models of the Gentzen calculus $\mathcal{G}_{\mathbf{W R}}$ (and are moreover finitary and have no theorems), calling them WR-logics. Among other results, we characterize the reduced WR-logics as the abstract logics $\mathbb{L}=\langle\mathfrak{A}, C\rangle$ such that $\mathfrak{A}$ is an $\mathbf{R}$-algebra and $C$ is the closure system of all the lattice filters of $\mathfrak{A}$ plus the empty set, and prove that in this case this closure system consists of exactly all the WR-filters on $\mathfrak{A}$. As a consequence we obtain that the $\mathbf{R}$-algebras can be characterized as the algebras where the abstract logic determined by the closure system of all the WR-filters is reduced, and that WRlogics are the inverse images of abstract logics of this form by bilogical morphisms. These results establish direct links between the deductive system WR and the class of WR-logics and enable us to draw some consequences using general results of [16]; probably the most interesting one is that on any algebra $\mathfrak{A}$ (of suitable similarity type) there is an isomorphism between the lattice of all WR-logics on it and the lattice of all congruences of the algebra whose quotient is an $\mathbf{R}$-algebra. 
In Section 5 we make a similar study for the deductive system $\mathbf{R}$. However, we do not have a structural Gentzen calculus giving exactly $\mathbf{R}$ in the standard way (Belnap's Display Logic, see section 62 of [2] incorporates in an essential way an additional negation with a classical behavior). Therefore the notion of $\mathbf{R}$-logic is first defined indirectly, as the class of axiomatic extensions of WR-logics by the set of all the "identity elements" $a \rightarrow a$. We prove that each WR-logic is in turn determined uniquely by the $\mathbf{R}$-logic it determines through this definition, and thus the lattices of all R-logics and of all WR-logics on a given algebra are isomorphic. The main results are parallel to those of Section 4: the reduced $\mathbf{R}$-logics have the form $\mathbb{L}=\langle\mathfrak{A}, C\rangle$ where $\mathfrak{A}$ is an $\mathbf{R}$-algebra, and $C$ is the closure system of the lattice filters of $\mathfrak{A}$ which contain all elements of the form $a \rightarrow a$, and general $\mathbf{R}$-logics are the inverse images of these by bilogical morphisms. Using the algebraizability of $\mathbf{R}$ we prove that $\mathbf{R}$-logics can be characterized as the abstract logics whose closure system consists of all the $\mathbf{R}$ filters that contain a given one, a fact that cannot be obtained for WR. We close this section with the characterization of $\mathbf{R}$-logics as those finitary models of $\mathbf{R}$ that satisfy a parametrically restricted form of the Deduction Theorem with respect to their own set of theorems.

2 The Deductive System $\mathbf{R}$ and its Algebraic Models Let $\mathfrak{F m}=\langle F m, \wedge, \rightarrow, \neg\rangle$ be the absolutely free algebra of type $(2,2,1)$ over a denumerable set of variables Var. We will denote as $\mathbf{R}=\left\langle\mathfrak{F m}, \vdash_{\mathbf{R}}\right\rangle$ a deductive system corresponding in a natural way to the well-known system $\mathbf{R}$ of Relevance Logic. The consequence relation $\vdash_{\mathbf{R}}$ is defined in the usual (Hilbert-style) form by means of the following list of axioms and inference rules (see [1], p. 341), where by definition

$$
\begin{aligned}
\varphi \vee \psi & =\neg(\neg \varphi \wedge \neg \psi) \\
\varphi \leftrightarrow \psi & =(\varphi \rightarrow \psi) \wedge(\psi \rightarrow \varphi) \\
\varphi * \psi & =\neg(\varphi \rightarrow \neg \psi)
\end{aligned}
$$

for any $\varphi, \psi \in F m$. The binary connective $*$ is commonly called intensional conjunction or fusion. The axioms of $\mathbf{R}$ are all formulas having one of the following forms:

$$
\begin{array}{ll}
\text { (R1) } & \varphi \rightarrow \varphi \\
\text { (R2) } & (\varphi \rightarrow \psi) \rightarrow((\psi \rightarrow \eta) \rightarrow(\varphi \rightarrow \eta)) \\
\text { (R3) } & \varphi \rightarrow((\varphi \rightarrow \psi) \rightarrow \psi) \\
\text { (R4) } & (\varphi \rightarrow(\varphi \rightarrow \psi)) \rightarrow(\varphi \rightarrow \psi) \\
\text { (R5) } & (\varphi \wedge \psi) \rightarrow \varphi \\
\text { (R6) } & (\varphi \wedge \psi) \rightarrow \psi \\
\text { (R7) } & ((\varphi \rightarrow \psi) \wedge(\varphi \rightarrow \eta)) \rightarrow(\varphi \rightarrow(\psi \wedge \eta)) \\
\text { (R8) } & \varphi \rightarrow(\varphi \vee \psi) \\
\text { (R9) } & \psi \rightarrow(\varphi \vee \psi) \\
\text { (R10) } & ((\varphi \rightarrow \eta) \wedge(\psi \rightarrow \eta)) \rightarrow((\varphi \vee \psi) \rightarrow \eta) \\
\text { (R11) } & (\varphi \wedge(\psi \vee \eta)) \rightarrow((\varphi \wedge \psi) \vee \eta) \\
\text { (R12) } & (\varphi \rightarrow \neg \psi) \rightarrow(\psi \rightarrow \neg \varphi) \\
\text { (R13) } & \neg \neg \varphi \rightarrow \varphi
\end{array}
$$

and the inference rules are: 
(Adjunction) $\varphi, \psi \vdash \varphi \wedge \psi$
(Modus Ponens) $\quad \varphi, \varphi \rightarrow \psi \vdash \psi$.

Note that from now on $\mathbf{R}$ or $\vdash_{\mathbf{R}}$ will denote the deducibility relation from premises obtained by this formal system; therefore we are taking these inference rules as rules of deducibility and not just as rules of proof of theorems from theorems.

At several points in this paper we will use some theorems of $\mathbf{R}$ which we reproduce here with the same numbering of [1], pp. 396-397; their proof can be found somewhere in this book:

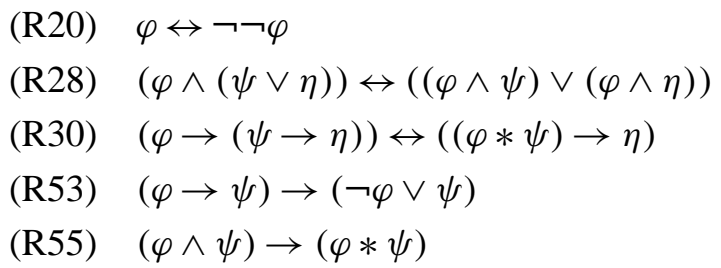

and we will also use the following two theorems of $\mathbf{R}$ :

$$
\begin{array}{ll}
\text { (WI) } & (\varphi \wedge(\varphi \rightarrow \psi)) \rightarrow \psi \\
\text { (RR) } & (((\varphi \rightarrow \varphi) \wedge(\psi \rightarrow \psi)) \rightarrow \eta) \rightarrow \eta .
\end{array}
$$

The first one is named after Slaney [24], and the second one is mentioned in [1] (p. 321) as a theorem of $\mathbf{E}$, of which $\mathbf{R}$ is a (nonconservative) extension. (RR) can actually substitute the characteristic modal axiom $(\square \varphi \wedge \square \psi) \rightarrow \square(\varphi \wedge \psi)$ of $\mathbf{E}$; as we shall see, this theorem (or better, its algebraic version) plays an essential role in the approach to the algebraization of $\mathbf{R}$ begun in 17.

The first algebraic models of $\mathbf{R}$, introduced in Dunn [14] (see also [1], §28.2) in order to study the algebraic completeness of $\mathbf{R}$, were called De Morgan semigroups. As a starting point we take the following alternative definition in terms of $\wedge, \neg, \rightarrow$, proved polynomially equivalent to the original in 17 , Theorem 5 modulo the definition of the semigroup operation $*$ given above: $a * b=\neg(a \rightarrow \neg b)$; in the converse process starting from $\wedge, \neg, *$, the implication is defined as $a \rightarrow b=\neg(a * \neg b)$.

Definition 2.1 An algebra $\mathfrak{A}=\langle A, \wedge, \rightarrow, \neg\rangle$ of type $(2,2,1)$ is a De Morgan semigroup when the following conditions hold:

(1) $\langle A, \wedge, \neg\rangle$ is a De Morgan lattice, whose ordering relation we denote by $\leq$, and whose supremum operation is precisely $a \vee b=\neg(\neg a \wedge \neg b)$.

(2) $a \rightarrow(b \rightarrow c) \leq b \rightarrow(a \rightarrow c)$, for any $a, b, c \in A$.

(3) $a \leq((a \rightarrow b) \wedge c) \rightarrow b$, for any $a, b, c \in A$.

(4) $a \rightarrow \neg a \leq \neg a$, for any $a \in A$.

(5) $a \rightarrow b \leq \neg b \rightarrow \neg a$, for any $a, b \in A$.

Recall that a De Morgan lattice is defined like a De Morgan algebra (see [3]) but without the condition that it must be bounded; so it need not have a smallest and a greatest element.

Dunn notes in [1], p. 361 that the variety of De Morgan semigroups is not a good enough class of algebraic models for $\mathbf{R}$. Indeed, one of the distinctive features of $\mathbf{R}$, which sets it apart from other nonclassical systems such as the intuitionistic one or 
Łukasiewicz's $\aleph_{0}$-valued system, is the fact that not every two theorems are equivalent; this is so because in $\mathbf{R}$ not all formulas $\varphi \rightarrow(\psi \rightarrow \varphi)$ are theorems. As a consequence, in general there is no distinguished element in De Morgan semigroups to interpret theorems. Moreover, the Tarski-Lindenbaum algebra of $\mathbf{R}, \mathfrak{F} \mathfrak{m} / \widetilde{\Omega}(\mathbf{R})$, where $\langle\varphi, \psi\rangle \in \widetilde{\Omega}(\mathbf{R}) \Longleftrightarrow \vdash_{\mathbf{R}} \varphi \leftrightarrow \psi$, is not free in the class of all De Morgan semigroups. Thus this class cannot give us the desired algebraic completeness result for R. Dunn's solution (see [1], pp. 361-366) consists in enlarging the language with a least truth value constant $\mathbf{t}$ and adding the formula $\mathbf{t}$ and the formula scheme $\mathbf{t} \rightarrow(\varphi \rightarrow \varphi)$ to the list of axioms of $\mathbf{R}$. The extended system $\mathbf{R}_{\mathbf{t}}$ thus obtained turns out to be a conservative extension of $\mathbf{R}$, and its algebraic models are De Morgan monoids, that is, De Morgan semigroups with a unit $e$. Since $\mathbf{R}_{\mathbf{t}}$ happens to be (strongly) complete with respect to this class of models, we can obtain as a byproduct a (strong) completeness result for $\mathbf{R}$ in the following form: for any $\Gamma \subseteq F m$ and any $\varphi \in F m, \Gamma \vdash_{\mathbf{R}} \varphi \Longleftrightarrow e \leq \varphi^{\mathfrak{A}}(\bar{a})$ for any interpretation $\bar{a}$ in any De Morgan monoid $\mathfrak{A}$ with unit $e$ such that $e \leq \psi^{\mathfrak{A}}(\bar{a})$ for all $\psi \in \Gamma$.

In 17 an alternative solution is presented. First of all, it is proved that De Morgan semigroups themselves provide us with a matrix semantics for $\mathbf{R}$ in a rather natural way. Recall that one says that a logical matrix $\langle\mathfrak{A}, F\rangle$ is an $\mathbf{R}$-matrix (or, equivalently, that $F$ is an $\mathbf{R}$-filter on $\mathfrak{A}$ ) when for any $\Gamma \cup\{\varphi\} \subseteq F m$, the relation $\Gamma \vdash_{\mathbf{R}} \varphi$ implies that for any interpretation $\bar{a}$ on $\mathfrak{A}$, if $\left\{\psi^{\mathfrak{A}}(\bar{a}): \psi \in \Gamma\right\} \subseteq F$ then $\varphi^{\mathfrak{A}}(\bar{a}) \in F$. The class of all $\mathbf{R}$-matrices will be denoted in this paper by MatrR, and for any algebra $\mathfrak{A}$, the set of all $\mathbf{R}$-filters on $\mathfrak{A}$ will be denoted by $\mathcal{F} i_{\mathbf{R}} \mathfrak{A}$. On the other hand, on any distributive lattice $\mathfrak{A}$ we will consider the following closure system associated with the family of all lattice filters of $\mathfrak{A}$ :

$$
\mathcal{F}_{\text {llt } \mathfrak{A}}=\{F \subseteq A: F \text { is a lattice filter of } \mathfrak{A} \text { or } F=\varnothing\},
$$

and we denote by Filt the closure operator associated with this closure system. Note the nonstandard character of this definition: actually, for any $X \subseteq A$, if $X \neq \varnothing$ then Filt $(X)$ is the lattice filter generated by $X$, whereas Filt $(\varnothing)=\varnothing$. Thus if the lattice has no greatest element (equivalently, if it has no least filter), then $\mathcal{F}_{\text {flt }} \mathfrak{A}$ is the closure system generated by the family of all lattice filters of $\mathfrak{A}$, whereas if $\mathfrak{A}$ has a greatest element then we have explicitly added the empty set. The reason for such an unusual procedure will become clear at the end of Section 4, namely in Theorem 4.14. If moreover $\mathfrak{A}$ is a De Morgan semigroup, we distinguish the filter generated by all elements of the form $a \rightarrow a$ by putting

$$
E(\mathfrak{A})=\operatorname{Filt}(\{a \rightarrow a: a \in A\})
$$

and consider the closure system of all deductive filters of $\mathfrak{A}$, namely $\operatorname{Ded} \mathfrak{A}=\{F \subseteq$ $A: F \in \mathcal{F}_{l} l t \mathfrak{A}$ and $\left.E(\mathfrak{A}) \subseteq F\right\}$, denoting by Ded its associated closure operator; this use of the term "deductive filter" is justified by the following result.

Theorem 2.2 (17)

(1) For every De Morgan semigroup $\mathfrak{A}, \mathcal{F} i \mathbf{R}_{\mathbf{R}} \mathfrak{A}=\mathcal{D e d} \mathfrak{A}$.

(2) $\mathbf{R}$ is (strongly) complete with respect to the class of matrices $\{\langle\mathfrak{A}, E(\mathfrak{A})\rangle: \mathfrak{A}$ is a De Morgan semigroup\}. 
The introduction in 17 of the set $E(\mathfrak{A})$ was motivated by an observation of Dunn to the effect that from any proof in $\mathbf{R}_{\mathbf{t}}$ of a formula without $\mathbf{t}$ one can obtain a proof of the same formula in $\mathbf{R}$ simply by substituting all occurrences of $\mathbf{t}$ by the conjunction of the formulas $p \rightarrow p$, where $p$ ranges over all variables appearing in the original proof. A detailed consideration of this fact and its algebraic consequences leads to a new class of algebraic structures:

Definition 2.3 (17]) An algebra $\mathfrak{A}=\langle A, \wedge, \rightarrow, \neg\rangle$, of type $(2,2,1)$ is an $\mathbf{R}$-algebra when $\mathfrak{A}$ is a De Morgan semigroup such that, for any $a, b, c \in A,((a \rightarrow a) \wedge(b \rightarrow$ $b)) \rightarrow c \leq c$. The variety of $\mathbf{R}$-algebras will be denoted by $\mathrm{R}$.

It is shown in [17] that $R$ is a proper subclass of the variety of De Morgan semigroups. As we have already noticed, its definition (together with all the properties these algebras will have) shows the importance of the theorem of $\mathbf{R}$ we have called $R R$. The following facts about the variety $R$ will be used in the paper:

Theorem 2.4 ([17)

(1) For any $\mathfrak{A} \in \mathrm{R}$ and any $a, b \in A, a \leq b \Longleftrightarrow a \rightarrow b \in E(\mathfrak{A})$.

(2) For any $\mathfrak{A} \in \mathrm{R}, \mathcal{F} i_{\mathbf{R}} \mathfrak{A}=\mathcal{D e d} \mathfrak{A}$.

(3) $\mathbf{R}$ is (strongly) complete with respect to the class of matrices $\{\langle\mathfrak{A}, E(\mathfrak{A})\rangle: \mathfrak{A} \in$ $\mathrm{R}\}$.

(4) For any $\varphi, \psi \in F m, \vdash_{\mathbf{R}} \varphi \rightarrow \psi \Longleftrightarrow \varphi^{\mathfrak{A}}(\bar{a}) \leq \psi^{\mathfrak{A}}(\bar{a})$ for any interpretation $\bar{a}$ on any $\mathfrak{A} \in \mathrm{R}$.

(5) $\mathrm{R}$ is the variety generated by the Tarski-Lindenbaum algebra $\mathfrak{F m} / \widetilde{\Omega}(\mathbf{R})$, which is the free algebra in $\mathrm{R}$.

On the other hand, after Theorem 5.8 of [5] we know that $\mathbf{R}$ is algebraizable in the precise sense of this term established in the same paper. This means that there is a unique quasivariety of algebras associated in a canonical form with $\mathbf{R}$, called its equivalent quasivariety semantics, which bears to $\mathbf{R}$ the closest relationship so far described between a logic and a class of algebras. The determination of this class, which in this case happens to be a variety, closes the problem of the algebraization of R.

Theorem 2.5 ([17]) The class $\mathrm{R}$ is the equivalent variety semantics for the deductive system $\mathbf{R}$, with defining equation $p \wedge(p \rightarrow p) \approx p \rightarrow p$ and equivalence formula $p \leftrightarrow q$.

This theorem, read under the light of the general study of algebraizability of deductive systems contained in [5], tells us that the class of algebras $\mathrm{R}$ is the algebraic counterpart of $\mathbf{R}$. It is easy to see that every De Morgan monoid is an $\mathbf{R}$ algebra, but the converse does not hold: take for instance the totally ordered set $A=([-1,0) \cup(0,1]) \cap \mathbb{Q}$ with the Sugihara operations (see [1], p. 400 or 421). Therefore, complementing the claim made in [2], p. 157 that “... we know after [1], $\S 28.2$ that De Morgan monoids are the right algebraic structure for $\mathbf{R}$," what we now know is that they are, actually, the best algebraic structures for $\mathbf{R}_{\mathbf{t}}$, in the same sense as $\mathbf{R}$-algebras are the best algebraic structures for $\mathbf{R}$.

We now recall some more notation and terminology in order to express some consequences of this fact and for the rest of the paper. For any algebra $\mathfrak{A}=\langle A, \wedge, \rightarrow$, 
$\neg)$ of type $(2,2,1)$, Con $\mathfrak{A}$ denotes the set of all congruences of $\mathfrak{A}$; and the congruences in $\operatorname{Con}_{R} \mathfrak{A}=\{\theta \in \operatorname{Con} \mathfrak{A}: \mathfrak{A} / \theta \in R\}$ are called the $\mathbf{R}$-congruences of $\mathfrak{A}$; if $\mathfrak{A} \in \mathrm{R}$ then $\operatorname{Con}_{\mathrm{R}} \mathfrak{A}=$ Con $\mathfrak{A}$ because $\mathrm{R}$ is a variety. On the other hand, for any $\mathfrak{A}=\langle A, \wedge, \rightarrow, \neg\rangle$ of type $(2,2,1)$ and any $F \subseteq A$ the congruences compatible with $F$ constitute the set $\operatorname{Con}\langle\mathfrak{A}, F\rangle=\{\theta \in \operatorname{Con} \mathfrak{A}:$ if $\langle a, b\rangle \in \theta$ then $a \in F \Longleftrightarrow b \in F\}$, and the Leibniz congruence of $F$ or of $\langle\mathfrak{A}, F\rangle$ is $\Omega_{\mathfrak{A}}(F)=\max \operatorname{Con}\langle\mathfrak{A}, F\rangle$. Then the mapping $\Omega_{\mathfrak{A}}: F \mapsto \Omega_{\mathfrak{A}}(F)$ restricted to $F \in \mathcal{F}_{i} \mathfrak{R}$ is called the Leibniz operator on $\mathfrak{A}$; this operator is one of the key tools in Blok and Pigozzi's study [5] of the algebraizability of logics. A matrix $\langle\mathfrak{A}, F\rangle$ is reduced when $\Omega_{\mathfrak{A}}(F)=\Delta_{A}$, the identity relation on $A$; the class of all reduced $\mathbf{R}$-matrices will be denoted by Matr* $\mathbf{R}$, and the class of the algebra reducts of these matrices will be denoted by RAlgR. Then from [5], Theorem 5.1 and Lemma 5.2, and [17], Theorem 18, we obtain:

\section{Theorem 2.6}

(1) On any algebra $\mathfrak{A}=\langle A, \wedge, \rightarrow, \neg\rangle$ of type $(2,2,1)$ the Leibniz operator $\Omega_{\mathfrak{A}}$ is an isomorphism between the lattices $\mathcal{F}_{\mathbf{R}} \mathfrak{A}$ and $\operatorname{Con}_{\mathrm{R}} \mathfrak{A}$, and moreover for any $F \in \mathcal{F} i_{\mathbf{R}} \mathfrak{A}$ we have that $\Omega_{\mathfrak{A}}(F)=\{\langle a, b\rangle \in A \times A: a \leftrightarrow b \in F\}$.

(2) $\operatorname{Matr}^{*} \mathbf{R}=\{\langle\mathfrak{A}, F\rangle: \mathfrak{A} \in \mathrm{R}$ and $F=E(\mathfrak{A})\}$.

(3) $R A \lg \mathbf{R}=R$.

Combining these results with Theorem 2.2 we also have:

Corollary 2.7 $\mathbf{R}$ is (strongly) complete with respect to the class of matrices $\{\langle\mathfrak{A}, F\rangle: \mathfrak{A} \in \mathrm{R}$ and $F \in \mathcal{D e d} \mathfrak{A}\}$.

These results close this brief overview of the relationships between the deductive system $\mathbf{R}$ and the variety of algebras $\mathbf{R}$ in the context of the traditional approach to the algebraization of logic — now greatly enhanced by Blok and Pigozzi's ideas and achievements. In Section 5 of the paper we will find other relationships between $\mathbf{R}$ and $\mathrm{R}$ using abstract logics.

3 The Weaker Deductive System WR and its Algebraic Models We are now going to define a deductive system WR following Wójcicki's suggestion mentioned in the Introduction; we also mentioned there that this deductive system corresponds to the semantic entailment associated with the relational models of Routley, Meyer, Fine and others. In this section we will study it along the lines of the study of $\mathbf{R}$ contained in 17 .

Definition 3.1 We call $\mathbf{W R}=\left\langle\mathfrak{F m}, \vdash_{\mathbf{W R}}\right\rangle$ the deductive system defined by the condition that, for any $\Gamma \cup\{\varphi\} \subseteq F m, \Gamma \vdash_{\mathbf{W R}} \varphi$ if and only if there are $\varphi_{1}, \ldots, \varphi_{n} \in$ $\Gamma$ such that $\vdash_{\mathbf{R}}\left(\varphi_{1} \wedge \ldots \wedge \varphi_{n}\right) \rightarrow \varphi$. This is the same as saying that $\mathbf{W R}$ is the deductive system determined by the following conditions:

(1) $\vdash \mathbf{W R}$ is finitary.

(2) WR has no theorems.

(3) For every $\varphi, \varphi_{1}, \ldots, \varphi_{n} \in F m,\left\{\varphi_{1}, \ldots, \varphi_{n}\right\} \vdash_{\mathbf{W R}} \varphi$ if and only if $\vdash_{\mathbf{R}}\left(\varphi_{1} \wedge\right.$ $\left.\ldots \wedge \varphi_{n}\right) \rightarrow \varphi$.

It is not difficult to show, by using some selected theorems of $\mathbf{R}$, that both ways of defining WR are really the same, and that in this way we really obtain a finitary 
and structural closure operator on $\mathfrak{F} \mathfrak{m}$, that is, a deductive system in the precise sense of the term. We are going to show that $\mathbf{W R}$ is weaker than $\mathbf{R}$, more precisely that $\mathbf{R}$ is an axiomatic extension of WR.

Lemma 3.2 The inference rules of $\mathbf{R}$, namely Adjunction and Modus Ponens, are also valid rules of the deductive system WR.

Proof: Axioms R5, R6, and the instance $\varphi \wedge \psi \rightarrow \varphi \wedge \psi$ of axiom R1 tell us directly that WR is closed under Adjunction (in the strong sense, that is, $\{\varphi, \psi\} \dashv \vdash_{\mathbf{R}} \varphi \wedge$ $\psi$ ). To see that it is closed under Modus Ponens (i.e., that $\{\varphi, \varphi \rightarrow \psi\} \vdash \mathbf{W R} \psi$ ) it is enough to use theorem WI mentioned in Section 2.

Theorem 3.3 The system $\mathbf{R}$ is the axiomatic extension of the system $\mathbf{W R}$ determined by all formulas of the form $\psi \rightarrow \psi$. That is, for any $\Gamma \cup\{\varphi\} \subseteq F m$ it holds that

$$
\Gamma \vdash_{\mathbf{R}} \varphi \Longleftrightarrow \Gamma \cup\{\psi \rightarrow \psi: \psi \in F m\} \vdash_{\mathbf{W R}} \varphi .
$$

Proof: Suppose that $\Gamma \vdash_{\mathbf{R}} \varphi$. If $\varphi \in \Gamma$ the conclusion is trivial. If $\varphi$ is an axiom of $\mathbf{R}$ (or, more generally, if $\vdash_{\mathbf{R}} \varphi$ ) then from R3 and Modus Ponens it follows that $\vdash_{\mathbf{R}}(\varphi \rightarrow \varphi) \rightarrow \varphi$, which by the definition of $\mathbf{W} \mathbf{R}$ implies the conclusion. Finally, if $\varphi$ is obtained from other formulas by Modus Ponens or Adjunction then Lemma 3.2 establishes the inductive step. Conversely, suppose that $\Gamma \cup\{\psi \rightarrow \psi: \psi \in F m\} \vdash \mathbf{W R}$ $\varphi$, that is, that there are $\varphi_{1}, \ldots, \varphi_{n} \in \Gamma$ and $\psi_{1}, \ldots, \psi_{k} \in F m$ such that $\vdash_{\mathbf{R}}\left(\varphi_{1} \wedge \ldots \wedge\right.$ $\left.\varphi_{n} \wedge\left(\psi_{1} \rightarrow \psi_{1}\right) \wedge \ldots \wedge\left(\psi_{k} \rightarrow \psi_{k}\right)\right) \rightarrow \varphi$; since all the $\psi_{i} \rightarrow \psi_{i}$ are axioms of $\mathbf{R}$, by Adjunction we have that $\varphi_{1}, \ldots, \varphi_{n} \vdash_{\mathbf{R}} \varphi_{1} \wedge \ldots \wedge \varphi_{n} \wedge\left(\psi_{1} \rightarrow \psi_{1}\right) \wedge \ldots \wedge\left(\psi_{k} \rightarrow\right.$ $\psi_{k}$ ), and then it follows by Modus Ponens that $\varphi_{1}, \ldots, \varphi_{n} \vdash_{\mathbf{R}} \varphi$, which proves that $\Gamma \vdash_{\mathbf{R}} \varphi$.

In particular we have that $\mathbf{W R} \supsetneqq \mathbf{R}$, that is, that $\Gamma \vdash_{\mathbf{W R}} \varphi$ implies $\Gamma \vdash_{\mathbf{R}} \varphi$, and that the converse implication does not hold. For $\Gamma=\varnothing$ this is so simply because WR has no theorems, but it does not hold even for nonempty $\Gamma$ : for as a particular case we would have $\psi \vdash_{\mathbf{W R}} \varphi \Longleftrightarrow \psi \vdash_{\mathbf{R}} \varphi$ for any $\varphi, \psi \in F m$; but since $\psi \rightarrow \psi$ is a theorem of $\mathbf{R}$, we also have $\psi \vdash_{\mathbf{R}} \psi \rightarrow \psi$, and thus we would also have $\psi \vdash \mathbf{W R}$ $\psi \rightarrow \psi$. By Definition 3 .1 his would mean that $\vdash_{\mathbf{R}} \psi \rightarrow(\psi \rightarrow \psi)$, which is known to be false (this is the well-known Mingle Axiom, which determines the system RM, a proper extension of $\mathbf{R}$ ).

Next we see that $R$ gives a matrix completeness result for $\mathbf{W R}$ in a very natural way, and from it we can characterize all WR-filters on the algebras in $R$.

Theorem 3.4 ((Completeness)) The logic WR is (strongly) complete with respect to the class of matrices $\left\{\langle\mathfrak{A}, F\rangle: \mathfrak{A} \in \mathrm{R}\right.$ and $\left.F \in \mathcal{F}_{\text {llt }} \mathfrak{A}\right\}$.

Proof: By Definition 3.13 we have that $\varphi \vdash_{\mathbf{W R}} \psi \Longleftrightarrow \vdash_{\mathbf{R}} \varphi \rightarrow \psi$, which by Theorem 2.4.4 holds if and only if $\varphi^{\mathfrak{A}}(\bar{a}) \leq \psi^{\mathfrak{A}}(\bar{a})$ for any interpretation $\bar{a}$ on any $\mathfrak{A} \in \mathrm{R}$, that is, if and only if for any $F \in \mathcal{F}_{l} l t \mathfrak{A}, \varphi^{\mathfrak{A}}(\bar{a}) \in F$ implies $\psi^{\mathfrak{A}}(\bar{a}) \in F$. By finitarity and Adjunction we obtain the completeness for nonempty sets of premises. Since WR does not have theorems, we should show that there is no formula whose interpretation belongs to every lattice filter of every $\mathbf{R}$-algebra, but this is obvious since there are $\mathbf{R}$-algebras (without greatest element) such that the intersection of all their 
lattice filters is empty; we will exhibit one such algebra, defined on the set of integers, just before Corollary 3.6 .

\section{Corollary 3.5 If $\mathfrak{A} \in \mathrm{R}$, then $\mathcal{F} \mathbf{W R}_{\mathbf{R}} \mathfrak{A}=\mathcal{F}_{\text {llt }} \mathfrak{A}$.}

Proof: $\quad$ The preceding theorem proves that $\mathcal{F}_{l l t} \mathfrak{A} \subseteq \mathcal{F}^{i} \mathbf{W R}^{\mathfrak{A}}$. Conversely, if $F \in$ ${ }^{\mathcal{F}} i_{\mathbf{W R}} \mathfrak{A}$ then by Lemma $3.2 F$ is closed under Adjunction, which implies that $a, b \in$ $F$ implies $a \wedge b \in F$; but it is also closed under Modus Ponens, and if $a \in F$ and $a \leq b$, that is, $a=a \wedge b$ then by R5 it follows that $b \in F$. Therefore $F \in \mathcal{F}_{7} l t \mathfrak{A}$, that is, $\mathcal{F}_{l} l t \mathfrak{A}=\mathcal{F} i_{\mathbf{W R}} \mathfrak{A}$.

As we recalled in Corollary 2.7. $\mathbf{R}$ is strongly complete with respect to the class of matrices $\{\langle\mathfrak{A}, F\rangle: \mathfrak{A} \in \mathrm{R}$ and $F \in \mathcal{D e d} \mathfrak{A}\}$ and for every $\mathfrak{A} \in \mathrm{R}, \mathcal{F} i_{\mathbf{R}} \mathfrak{A}=\operatorname{Ded} \mathfrak{A}$. In the case of $\mathbf{R}$ we can substitute the class of De Morgan semigroups for $R$ in these two results (see Theorem 2.2). However, we cannot do the same in the case of WR, that is, in Theorem 3.4 and Corollary 3.5. Actually, for every De Morgan semigroup $\mathfrak{A}$ and every $F \subseteq A$, if $\langle\mathfrak{A}, F\rangle$ is a WR-matrix, then it follows that $F \in \mathcal{F}_{\text {llt }} \mathfrak{A}$ by arguments similar to those used at the beginning of the proof of Theorem 3.4 (essentially concerning Adjunction); however, the converse implication does not hold, as the following example shows. Consider the four-element algebra whose lattice structure is given by the Hasse diagram below, and negation and implication are also shown below:

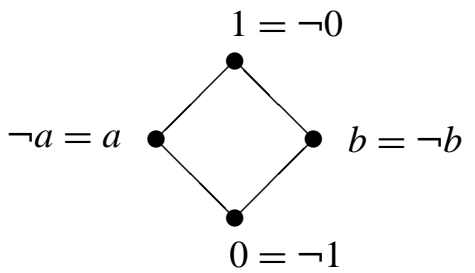

\begin{tabular}{c|cccc}
$\rightarrow$ & 0 & $a$ & $b$ & 1 \\
\hline 0 & 1 & 1 & 1 & 1 \\
$a$ & 0 & $a$ & 0 & 1 \\
$b$ & 0 & 0 & $b$ & 1 \\
1 & 0 & 0 & 0 & 1
\end{tabular}

This is a De Morgan semigroup which is not an $\mathbf{R}$-algebra. Take $F=\{a, 1\}$; this is a lattice filter of $\mathfrak{A}$, but the matrix $\langle\mathfrak{A}, F\rangle$ is not a WR-matrix because by RR $((p \rightarrow p) \wedge(q \rightarrow q)) \rightarrow r \vdash \mathbf{W R} r$ for any distinct $p, q, r \in \operatorname{Var}$, and while $((a \rightarrow$ $a) \wedge(b \rightarrow b)) \rightarrow b=1 \in F$ we have $b \notin F$.

We have already seen that WR differs from $\mathbf{R}$ not only in not having theorems, but in having different deductions from nonempty sets of hypotheses. Another interesting thing to see is that there is no formula which, while not being a theorem of WR, is a member of every nonempty theory. This is so for the same reason required for the last part of the proof of Theorem 3.4. such a formula would belong to every lattice filter of every $\mathbf{R}$-algebra, a situation which cannot happen, as the following example shows. Take the distributive lattice determined by the usual ordering on the set $\mathbb{Z}$ of integers, define negation as $\neg n=-n$ and implication as

$$
n \rightarrow m= \begin{cases}\max \{-n, m\} & \text { if } n \leq m \\ \min \{-n, m\} & \text { otherwise }\end{cases}
$$

and this determines a structure of $\mathbf{R}$-algebra on $\mathbb{Z}$ without smallest or greatest element and whose lattice filters are all the end segments $[z, \rightarrow)$ with $z \in \mathbb{Z}$; their intersection is obviously empty. Using the term pseudoaxiomatic introduced in [27], p. 382, we have proved: 
Corollary 3.6 WR is not pseudoaxiomatic. Moreover, WR is also the deductive system defined from $\mathbf{R}$ by Conditions (1) and (3) of Definition 3.1 and with condition

$\left(2^{\prime}\right) \vdash \mathbf{W R}$ is not pseudoaxiomatic, that is, the intersection of all its nonempty theories equals its set of theorems.

instead of Condition (2).

Proof: Since WR has no theorems, the preceding discussion shows that WR satisfies $\left(2^{\prime}\right)$. Conversely, we have to show that if WR is defined with $\left(2^{\prime}\right)$ instead of (2) then it also satisfies (2), that is, it has no theorems. But by $\left(2^{\prime}\right)$ this amounts to showing that there is no formula belonging to every nonempty theory. Now by (1) and (3) the nonempty theories are the same in both cases, so the same argument as before implies that there is no such formula.

Thus we find that WR can equally be defined by following the second approach of [27]; more precisely, we have proved that WR is the deductive system determined by the set of all theorems of $\mathbf{R}$ in the sense of [27], § 2.10.1. The fact that our logic has no theorems puts it automatically outside the class of protoalgebraic logics introduced in [4], and a fortiori outside the class of algebraizable logics introduced in [5; this fact has also been noticed in [9].

Proposition 3.7 WR is not a protoalgebraic logic and it is not algebraizable.

Proof: By the result in Czelakowski and Dziobiak [10 the only protoalgebraic deductive systems without theorems are the inconsistent one and the so-called almost inconsistent, whose only theories are the empty set and the whole set of formulas. But the set of theorems of $\mathbf{R}$ is a nonempty and proper theory of $\mathbf{W R}$, so this is not inconsistent nor almost inconsistent. As a consequence it cannot be protoalgebraic. And since every algebraizable deductive system is also protoalgebraic, it follows that WR is not algebraizable either.

This means that if we want to determine the class Matr*WR of all reduced matrices for WR, and the class RAlgWR of their algebra reducts, we cannot rely upon the fact that for algebraizable logics this last class equals the equivalent quasivariety semantics (as is done in 17 for $\mathbf{R}$ ), and moreover we have no a priori guarantee that the class RAlgWR is the class of algebras corresponding to the most natural models for WR. We are going to determine these classes of algebras and matrices in a direct way, and in the next section we will justify the last statement by different arguments.

Theorem 3.8 The class of algebra reducts of reduced matrices for $\mathbf{W R}$ is the class of $\mathbf{R}$-algebras; that is, $\mathbf{R A l g} \mathbf{W R}=\mathbf{R}$.

Proof: Since $\mathbf{R}$ is an (axiomatic) extension of $\mathbf{W R}$ we obviously have RAlgR $\subseteq$ RAlgWR, and by Theorem 2.6this implies that $R \subseteq$ RAlgWR. Conversely, suppose that $\mathfrak{A} \in \mathbf{R A l g} \mathbf{W R}$, that is, $\langle\mathfrak{A}, F\rangle \in \operatorname{Matr}^{*} \mathbf{W R}$ for some $F \subseteq A$. Since $\mathrm{R}$ is a variety it is enough to show that $\mathfrak{A}$ validates all equations true in $\mathrm{R}$. Let $\varphi, \psi \in F m$ be such that $\models_{\mathrm{R}} \varphi \approx \psi$ and take any $\gamma\left(p, p_{1}, \ldots, p_{n}\right) \in F m$, where $p, p_{1}, \ldots, p_{n} \in$ Var. The properties of equality imply that also $\models_{\mathrm{R}} \gamma\left(\varphi, p_{1}, \ldots, p_{n}\right) \approx \gamma\left(\psi, p_{1}, \ldots, p_{n}\right)$, the algebraizability of $\mathbf{R}$ (with respect to $\mathrm{R}$ and with equivalence formula $p \leftrightarrow q$ ) implies that this is equivalent to $\vdash_{\mathbf{R}} \gamma\left(\varphi, p_{1}, \ldots, p_{1}\right) \leftrightarrow \gamma\left(\psi, p_{1}, \ldots, p_{n}\right)$, and Condition (3) in Definition 3.1 implies that this is equivalent to $\gamma\left(\varphi, p_{1}, \ldots, p_{n}\right) \dashv \vdash_{\mathbf{W R}}$ 
$\gamma\left(\psi, p_{1}, \ldots, p_{n}\right)$. Thus, for any interpretation $\bar{a}$ on $\mathfrak{A}$ we have that $\gamma^{\mathfrak{A}}\left(\varphi^{\mathfrak{A}}(\bar{a})\right.$, $\left.p_{1}^{\mathfrak{A}}(\bar{a}), \ldots, p_{n}^{\mathfrak{A}}(\bar{a})\right) \in F \Longleftrightarrow \gamma^{\mathfrak{A}}\left(\psi^{\mathfrak{A}}(\bar{a}), p_{1}^{\mathfrak{A}}(\bar{a}), \ldots, p_{n}^{\mathfrak{A}}(\bar{a})\right) \in F$, which means that for any $c_{1}, \ldots, c_{n} \in A, \gamma^{\mathfrak{A}}\left(\varphi^{\mathfrak{A}}(\bar{a}), c_{1}, \ldots, c_{n}\right) \in F \Longleftrightarrow \gamma^{\mathfrak{A}}\left(\psi^{\mathfrak{A}}(\bar{a}), c_{1}, \ldots\right.$, $\left.c_{n}\right) \in F$. But this is an equivalent characterization of the Leibniz congruence of the matrix $\langle\mathfrak{A}, F\rangle$, see for instance 5, p. 11. Therefore we have proved that $\left\langle\varphi^{\mathfrak{A}}(\bar{a})\right.$, $\left.\psi^{\mathfrak{A}}(\bar{a})\right\rangle \in \Omega_{\mathfrak{A}}(F)$. But $\langle\mathfrak{A}, F\rangle$ is reduced, that is, $\Omega_{\mathfrak{A}}(F)=\Delta_{A}$, and then $\varphi^{\mathfrak{A}}(\bar{a})=$ $\psi^{\mathfrak{A}}(\bar{a})$. We have shown that $\varphi \approx \psi$ holds in $\mathfrak{A}$; thus, $\mathfrak{A} \in \mathrm{R}$.

We finally find the class Matr*WR of reduced matrices for WR by using the properties of the Leibniz operator on algebras in $R$ recalled in Theorem 2.6.

Theorem 3.9 $\langle\mathfrak{A}, F\rangle \in$ Matr*WR if and only if $\mathfrak{A} \in \mathrm{R}$ and $F \in \mathcal{F}_{\text {llt }} \mathfrak{A}$ is such that for every $T \in \mathcal{D e d} \mathfrak{A} \backslash\{E(\mathfrak{A})\}$ there are elements $a \in F$ and $b \in A \backslash F$ such that $a \leftrightarrow b \in T$.

Proof: If $\langle\mathfrak{A}, F\rangle \in$ Matr* WR we have just proved that $\mathfrak{A} \in \mathrm{R}$ and by Corollary 3.5 $F \in \mathcal{F}_{\text {llt }} \mathfrak{A}$. Now suppose that there is some $T \in \mathcal{D e d} \mathfrak{A} \backslash\{E(\mathfrak{A})\}$ such that for all $a \in F$ and $b \notin F, a \leftrightarrow b \notin T$; by Theorem $2.2 T$ is an $\mathbf{R}$-filter, and by Theorem 2.6.1 this implies that $\langle a, b\rangle \notin \Omega_{\mathfrak{A}}(T)$; in other words, $\Omega_{\mathfrak{A}}(T)$ is compatible with $F$ and thus $\Omega_{\mathfrak{A}}(T) \subseteq \Omega_{\mathfrak{A}}(F)$. Since $\langle\mathfrak{A}, F\rangle$ is reduced we conclude that $\Omega_{\mathfrak{A}}(T)=\Delta_{A}=$ $\Omega_{\mathfrak{A}}(E(\mathfrak{A}))$ and, by the isomorphism of Theorem 2.61 we obtain $T=E(\mathfrak{A})$ against the assumption on $T$. Conversely, note that the condition on $F$ implies that for any $T \in \operatorname{Ded} \mathfrak{A} \backslash\{E(\mathfrak{A})\}, \Omega_{\mathfrak{A}}(T) \neq \Omega_{\mathfrak{A}}(F)$; but since $\Omega_{\mathfrak{A}}(F) \in$ Con $\mathfrak{A}=$ Con $_{\mathrm{R}} \mathfrak{A}$ (because $\mathfrak{A} \in \mathrm{R}$ which is a variety), the isomorphism in Theorem 2.6.1 implies that it must be equal to $\Omega_{\mathfrak{A}}(E(\mathfrak{A}))$, which is the identity. Therefore, the matrix is reduced and $\langle\mathfrak{A}, F\rangle \in \operatorname{Matr}^{*} \mathbf{W R}$.

Thus, although for $\mathbf{R}$ and $\mathbf{W R}$ the classes of algebra reducts of reduced matrices are the same, this is not so for the reduced matrices themselves, since by Theorem 2.6.2 the reduced matrices for $\mathbf{R}$ are those of the form $\langle\mathfrak{A}, E(\mathfrak{A})\rangle$ for some $\mathfrak{A} \in \mathrm{R}$. That these classes are different is confirmed by the following example. Consider the structure determined on the five-element chain $\{0, a, b, \neg a, 1\}$ by the negation and implication shown at the top of the next page.

It is easy to check that this is an $\mathbf{R}$-algebra and that $E(\mathfrak{A})=\{b, \neg a, 1\}$. Now take $F=\{\neg a, 1\}$ and check that $\langle\mathfrak{A}, F\rangle \in \operatorname{Matr}^{*} \mathbf{W R}$ : on the one hand $\langle\mathfrak{A}, F\rangle \in$ MatrWR because $F \in \mathcal{F}_{\text {llt }} \mathfrak{A}$; on the other hand, since $\mathcal{F} i \mathbf{R}^{\mathfrak{A}}=\operatorname{Ded} \mathfrak{A}=\{E(\mathfrak{A}), T, A\}$ where $T=\{a, b, \neg a, 1\}$, we know by Theorem 2.6 that Con $\mathfrak{A}=\left\{\Delta_{A}\right.$, $\left.\Omega_{\mathfrak{A}}(T), A \times A\right\}$. But $\Omega_{\mathfrak{A}}(T)$ is not compatible with $F$, because $\neg a \rightarrow a=a \in T$ and $a \rightarrow \neg a=\neg a \in T$ while $\neg a \in F$ but $a \notin F$. This shows that $\Omega_{\mathfrak{A}}(F)=\Delta_{\mathfrak{A}}$, that is, $\langle\mathfrak{A}, F\rangle \in$ Matr* $^{*} \mathbf{R}$; and it also exhibits the elements that satisfy the condition in Theorem 3.9 for this $T$.

4 A Gentzen Calculus for WR, and its Models The deductive system WR has been introduced so far with the auxiliary help of the deductive system $\mathbf{R}$, of which it is undoubtedly a by-product. But since we want to consider it as formally representing a certain kind of implication or entailment, we believe it deserves a definition of its own, a so to speak direct one. We will give it in the form of a sequent calculus. For our purposes a sequent is an ordered pair $\langle\Gamma, \varphi\rangle$, where $\Gamma \subseteq F m$ is finite and nonempty 


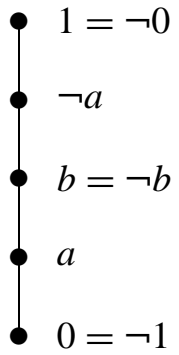

\begin{tabular}{c|ccccc}
$\rightarrow$ & 0 & $a$ & $b$ & $\neg a$ & 1 \\
\hline 0 & 1 & 1 & 1 & 1 & 1 \\
$a$ & 0 & $\neg a$ & $\neg a$ & $\neg a$ & 1 \\
$b$ & 0 & $a$ & $b$ & $\neg a$ & 1 \\
$\neg a$ & 0 & $a$ & $a$ & $\neg a$ & 1 \\
1 & 0 & 0 & 0 & 0 & 1
\end{tabular}

(!) and $\varphi \in F m$; we will represent it as $\Gamma \vdash \varphi$ for traditional reasons. The set of all these sequents will be denoted by $\operatorname{Seq}^{*}(\mathfrak{F m})$. All sequents appearing in this paper are assumed to belong to this set; in dealing with sequents we use the customary abbreviations: $\varphi$ for $\{\varphi\}, \Gamma, \varphi$ for $\Gamma \cup\{\varphi\}$ and so on.

Definition 4.1 By $\mathcal{G}_{\mathbf{W R}}=\left\langle\mathfrak{F m}, \sim_{\mathcal{G}_{\mathbf{W R}}}\right\rangle$ we denote the Gentzen calculus associated with the finitary and structural closure operator $\sim_{G}{ }_{\mathbf{W R}}$ over Seq*(Fm) defined by the following axioms:

(1) $\varphi \vdash \varphi$

(2) $\varphi \rightarrow(\psi \rightarrow \eta) \vdash \psi \rightarrow(\varphi \rightarrow \eta)$

(3) $((\varphi \rightarrow \varphi) \wedge(\psi \rightarrow \psi)) \rightarrow \eta \vdash \eta$

and the following Gentzen rules (taken as rules of inference from sequents to sequents):

$$
\begin{aligned}
& \text { (Weakening) } \frac{\Gamma \vdash \varphi}{\Gamma, \psi \vdash \varphi} \\
& \text { (Cut) } \frac{\Gamma \vdash \varphi \quad \Gamma, \varphi \vdash \psi}{\Gamma \vdash \psi} \\
& (\wedge \vdash) \frac{\Gamma, \varphi, \psi \vdash \eta}{\Gamma, \varphi \wedge \psi \vdash \eta} \\
& (\vdash \wedge) \frac{\Gamma \vdash \varphi \quad \Gamma \vdash \psi}{\Gamma \vdash \varphi \wedge \psi} \\
& (\vee \vdash) \frac{\Gamma, \varphi \vdash \eta \quad \Gamma, \psi \vdash \eta}{\Gamma, \varphi \vee \psi \vdash \eta} \\
& (\vdash \vee) \frac{\Gamma \vdash \varphi}{\Gamma \vdash \varphi \vee \psi} \quad \frac{\Gamma \vdash \psi}{\Gamma \vdash \varphi \vee \psi} \\
& \text { (ᄀ) } \frac{\varphi \vdash \psi}{\neg \psi \vdash \neg \varphi} \\
& (\neg \neg \vdash) \frac{\Gamma, \varphi \vdash \psi}{\Gamma, \neg \neg \varphi \vdash \psi} \\
& (\vdash \neg \neg) \frac{\Gamma \vdash \varphi}{\Gamma \vdash \neg \neg \varphi} \\
& (\rightarrow \vdash) \frac{\Gamma \vdash \varphi}{\Gamma, \varphi \rightarrow \psi \vdash \psi} \\
& \text { (*1) } \frac{\varphi * \psi \vdash \eta}{\varphi \vdash \psi \rightarrow \eta} \\
& \text { (*2) } \frac{\varphi \vdash \psi \rightarrow \eta}{\varphi * \psi \vdash \eta .}
\end{aligned}
$$

If $\Xi$ is a set of sequents then we write $\Xi \sim_{\mathcal{G}} \mathbf{W R}, \Gamma \vdash \varphi$ to mean that the sequent $\Gamma \vdash \varphi$ has a derivation in this calculus whose initial sequents are either axioms or belong to the set $\boldsymbol{\Xi}$; if $\varnothing \sim_{\mathcal{G}_{\mathbf{W R}}} \Gamma \vdash \varphi$ then we say that the sequent $\Gamma \vdash \varphi$ is derivable in $\mathcal{G}_{\mathbf{W R}}$.

Note that we have not explicitly included the rules of exchange and contraction among the rules of the calculus because they are automatically valid since we are using sets of formulas in the sequents, not just multisets or sequences. Thus this sequent calculus satisfies all structural rules, which is not common among the Gentzen-style 
presentations of relevance logics (see Došen [12] and Ono [23]); but recall that we are interested just in defining the deductive system $\mathbf{W R}$, and as such it must satisfy the structural rules by definition. We can associate a deductive system with this Gentzen calculus in a natural way:

Definition 4.2 $\mathcal{S}_{\mathbf{W R}}=\left\langle\mathfrak{F m}, \vdash_{G_{\mathbf{W R}}}\right\rangle$ is the deductive system defined in the following way: for any $\Gamma \cup\{\varphi\} \subseteq F m, \Gamma \vdash_{G} \mathbf{W R}_{\mathbf{R}} \varphi \Longleftrightarrow$ there is a finite $\Gamma_{0} \subseteq \Gamma$ such that $\Gamma_{0} \vdash \varphi$ is a sequent derivable in $\mathcal{G}_{\mathbf{W R}}$.

It is straightforward to check that $\mathcal{S}_{\mathbf{W R}}$ is indeed a deductive system (actually this definition works for every Gentzen system with all structural rules, see [16, $\S 4.1$ ) and that it has no theorems. Our aim is to prove that this is another, independent, presentation of the logic $\mathbf{W R}$, that is, that $\mathcal{S}_{\mathbf{W R}}=\mathbf{W R}$ as deductive systems. To this end we will study the models of this sequent calculus following the notion of model of a sequent calculus introduced in 15, Definition 3; later on we will see that a particular class of these models is also related to the abstract study of system $\mathbf{R}$. The mathematical objects we take to speak about models of a Gentzen calculus are the abstract logics introduced in [7]. An abstract logic is an ordered pair $\mathbb{L}=\langle\mathfrak{A}, C\rangle$, where $\mathfrak{A}$ is an abstract algebra with universe $A$ and $\mathrm{C}$ is a closure operator over $A$ (i.e., over its power set); they can also be presented as ordered pairs $\mathbb{L}=\langle\mathfrak{A}, C\rangle$, where $C$ is the closure system associated with the closure operator $\mathrm{C}$. An ordering relation can be considered between abstract logics having the same universe: $\mathbb{L}=\langle\mathfrak{A}, \mathrm{C}\rangle \leq \mathbb{L}^{\prime}=\left\langle\mathfrak{A}, \mathrm{C}^{\prime}\right\rangle$ if and only if for every $X \subseteq A, \mathrm{C}(X) \subseteq \mathrm{C}^{\prime}(X)$; in terms of the corresponding closure systems this amounts to the reverse inclusion $C^{\prime} \subseteq C$.

Definition 4.3 Let $\mathbb{L}=\langle\mathfrak{A}, \mathrm{C}\rangle$ be an abstract logic of type $(2,2,1)$, where $\mathfrak{A}=$ $\langle A, \wedge, \rightarrow, \neg\rangle$. We say that $\mathbb{L}$ is a model of $\mathcal{G}_{\mathbf{W R}}$ when for any rule of the form $\frac{\left\{\Gamma_{i} \vdash \varphi_{i}: i \in I\right\}}{\Gamma \vdash \varphi}$ where $I$ is not necessarily nonempty, if the rule is valid in $\mathcal{G}_{\mathbf{W R}}$ (that is, if the sequent $\Gamma \vdash \varphi$ has a derivation in $\mathcal{G}_{\mathbf{W R}}$ whose initial sequents are either axioms or belong to $\left\{\Gamma_{i} \vdash \varphi_{i}: i \in I\right\}$ ), then for any interpretation $\bar{a}$ over $\mathfrak{A}$, if $\varphi_{i}^{\mathfrak{A}}(\bar{a}) \in \mathrm{C}\left(\left\{\psi^{\mathfrak{A}}(\bar{a}): \psi \in \Gamma_{i}\right\}\right)$ for all $i \in I$ then $\varphi^{\mathfrak{A}}(\bar{a}) \in \mathrm{C}\left(\left\{\psi^{\mathfrak{A}}(\bar{a}): \psi \in \Gamma\right\}\right)$.

By its own definition it is clear that the deductive system $\mathcal{S}_{\mathbf{W R}}$ is one of the models of $\mathcal{G}_{\mathbf{W R}}$, and moreover it has two additional properties which are not inherited by all models, namely it is finitary and has no theorems. Since these are also key properties of WR and we want to study some abstract properties of this deductive system, we select from arbitrary models those satisfying them:

Definition 4.4 An abstract logic $\mathbb{L}=\langle\mathfrak{A}, \mathrm{C}\rangle$, of type $(2,2,1)$ will be called a WRlogic if and only if it is a finitary model of $\mathcal{G}_{\mathbf{W R}}$ such that $\mathrm{C}(\varnothing)=\varnothing$. For any algebra $\mathfrak{A}=\langle A, \wedge, \rightarrow, \neg\rangle$ of the same type we will denote by $\mathbb{W} \mathbb{R}(\mathfrak{A})$ the class of all WRlogics over $\mathfrak{A}$.

Thus $\mathcal{S}_{\mathbf{W R}}$ is one of the $\mathbf{W R}$-logics on the formula algebra $\mathfrak{F m}$, and indeed we will shortly prove that it is the least WR-logic on this algebra. We first give a straightforward characterization of WR-logics in terms of an already known kind of abstract logic. If $\mathbb{L}=\langle\mathfrak{A}, \mathrm{C}\rangle$ is an abstract logic of type $(2,1)$, where $\mathfrak{A}=\langle A, \wedge, \neg\rangle$ and we put $a \vee b=\neg(\neg a \wedge \neg b)$, then we say (see [18], 19]) that it is a De Morgan logic if and only if it satisfies the following conditions: 
(DM1) $\mathrm{C}$ is finitary;

(DM2) $\forall a, b \in A, \mathrm{C}(a, b)=\mathrm{C}(a \wedge b)$ (Conjunction);

(DM3) $\forall a, b \in A, \forall X \subseteq A, \mathrm{C}(X, a \vee b)=C(X, a) \cap \mathrm{C}(X, b)$ (Disjunction);

(DM4) $\forall a \in A, \mathrm{C}(a)=\mathrm{C}(\neg \neg a)$;

(DM5) $\forall a, b \in A, b \in \mathrm{C}(a) \Longrightarrow \neg a \in \mathrm{C}(\neg b)$.

This definition is shown in [19], Proposition 1 to be equivalent to the definition used in 18], and thus all the results found in this last paper apply to it. In terms of this notion we can immediately prove, by direct inspection of Definition 4.1, the following characterization:

Proposition 4.5 Let $\mathbb{L}=\langle\mathfrak{A}, \mathrm{C}\rangle$ be an abstract logic, where $\mathfrak{A}=\langle A, \wedge, \rightarrow, \neg\rangle$ is of type $(2,2,1)$. Then $\mathbb{L} \in \mathbb{W} \mathbb{R}(\mathfrak{A})$ if and only if $\mathbb{L}$ fulfills the following conditions:

(1) The abstract logic $\langle\langle A, \wedge, \neg\rangle, \mathrm{C}\rangle$ is a De Morgan logic such that $\mathrm{C}(\varnothing)=\varnothing$;

(2) $\forall a, b \in A, b \in \mathrm{C}(a, a \rightarrow b)$;

(3) $\forall a, b, c \in A, c \in \mathrm{C}(a * b) \Longleftrightarrow b \rightarrow c \in \mathrm{C}(a)$;

(4) $\forall a, b, c \in A, b \rightarrow(a \rightarrow c) \in \mathrm{C}(a \rightarrow(b \rightarrow c))$;

(5) $\forall a, b, c \in A, c \in \mathrm{C}(((a \rightarrow a) \wedge(b \rightarrow b)) \rightarrow c)$.

Now we can describe precisely the least WR-logic on several particular algebras. Recall that in Section 2 we have introduced, for a lattice $\mathfrak{A}$, the notation $\mathcal{F}_{\text {llt }} \mathfrak{A}$ for the closure system of all lattice filters of $\mathfrak{A}$ plus the empty set, and the notation Filt for the associated closure operator.

Theorem 4.6 For any $\mathfrak{A} \in \mathrm{R}$ the abstract logic $\langle\mathfrak{A}, \mathcal{F}$ llt $\mathfrak{A}\rangle$ is the least WR-logic on $\mathfrak{A}$.

Proof: Assume that $\mathfrak{A} \in \mathrm{R}$. We first prove that the abstract logic $\left\langle\mathfrak{A}, \mathcal{F}_{l} l t \mathfrak{A}\right\rangle$ fulfills the five conditions in the preceding proposition. Since by Definitions 2.1 and 2.3 we know that $\langle A, \wedge, \neg\rangle$ is a De Morgan lattice, then by [18], Theorem 3 the abstract $\operatorname{logic}\left\langle\langle A, \wedge, \neg\rangle, \mathcal{F}_{\text {llt }} \mathfrak{A}\right\rangle$ is a De Morgan logic such that $a \leq b \Longleftrightarrow b \in \operatorname{Filt}(a)$. Moreover by its own definition Filt $(\varnothing)=\varnothing$, so we have 4.5.1. Using the just mentioned equivalence, Conditions 4.5,2, 4.5.3 and 4.5.4 follow from properties P9, P1 and P11 recorded in 17], Propositions 4 and 2, and Condition 4.5.5 follows from Definition 2.3. Thus $\left\langle\mathfrak{A}, \mathcal{F}_{\text {fllt }} \mathfrak{A}\right\rangle$ is a WR-logic over $\mathfrak{A}$. Now any other WR-logic over $\mathfrak{A}$ will in particular be a De Morgan logic (after deletion of $\rightarrow$ from the type) and by Theorem 4 and Proposition 5 of [18] its closed sets will all be lattice filters of $\mathfrak{A}$; since neither can have theorems we conclude that $\left\langle\mathfrak{A}, \mathcal{F}_{l} l t \mathfrak{A}\right\rangle$ will have more closed sets, that is, it will be smaller.

Theorem 4.7 The deductive system $\mathcal{S}_{\mathbf{W R}}$ is the least $\mathbf{W R}$-logic over the formula algebra $\mathfrak{F m}$.

Proof: We have already commented that by its own definition $\mathcal{S}_{\mathbf{W R}} \in \mathbb{W} \mathbb{R}(\mathfrak{F m})$. Now take any $\mathbb{L}=\langle\mathfrak{F m}, \mathrm{C}\rangle \in \mathbb{W} \mathbb{R}(\mathfrak{F m})$; we must show that $\mathcal{S}_{\mathbf{W R}} \leq \mathbb{L}$, that is, that if $\Gamma \cup\{\varphi\} \subseteq F m$ and $\Gamma \vdash_{G_{\mathbf{W R}}} \varphi$ then $\varphi \in \mathrm{C}(\Gamma)$. But $\Gamma \vdash_{G_{\mathbf{W R}}} \varphi$ if and only if there is a finite and nonempty $\Gamma_{0} \subseteq \Gamma$ such that the sequent $\Gamma_{0} \vdash \varphi$ is derivable in $\mathcal{G}_{\mathbf{W R}}$. Then a straightforward induction on the length of this derivation using the very definition of being a model of $\mathcal{G}_{\mathbf{W R}}$ proves that $\varphi \in \mathrm{C}\left(\Gamma_{0}\right) \subseteq \mathrm{C}(\Gamma)$, that is, that $\mathcal{S}_{\mathbf{W R}} \leq \mathbb{L}$. 
To prove the identity between $\mathcal{S}_{\mathbf{W R}}$ and $\mathbf{W R}$ we will first prove some technical properties of WR-logics depending on the characterization of Proposition 4.5. Some of them tell us about properties of the closed set of $C$ generated by the elements of the universe having the form $a \rightarrow a$ for $a \in A$; this closed set is the abstract counterpart of the lattice filter $E(\mathfrak{A})$ we have considered on $\mathbf{R}$-algebras. Thus, for any abstract logic $\mathbb{L}=\langle\mathfrak{A}, \mathrm{C}\rangle$ of type $(2,2,1)$ it seems reasonable to define $E(\mathbb{L})=\mathrm{C}(\{a \rightarrow a: a \in A\})$. We then have the following facts, the last two being the most significant:

Proposition 4.8 If $\mathbb{L}=\langle\mathfrak{A}, \mathrm{C}\rangle \in \mathbb{W} \mathbb{R}(\mathfrak{A})$ then $\forall a, b, c \in A$ the following hold:

(1) $\mathrm{C}(a) \subseteq \mathrm{C}(b) \Longrightarrow \mathrm{C}(a * c) \subseteq \mathrm{C}(b * c)$ and $\mathrm{C}(c \rightarrow a) \subseteq \mathrm{C}(c \rightarrow b)$;

(2) $\mathrm{C}(a \rightarrow b)=\mathrm{C}(\neg(a * \neg b))$;

(3) $\mathrm{C}(a) \subseteq \mathrm{C}(b) \Longrightarrow \mathrm{C}(c * a) \subseteq \mathrm{C}(c * b)$ and $\mathrm{C}(b \rightarrow c) \subseteq \mathrm{C}(a \rightarrow c)$;

(4) $b \in \mathrm{C}(a) \Longleftrightarrow a \rightarrow b \in E(\mathbb{L})$;

(5) $\mathrm{C}(a * b)=\mathrm{C}(b * a)$;

(6) $(b \rightarrow c) \rightarrow(a \rightarrow c) \in \mathrm{C}(a \rightarrow b)$;

(7) $a \rightarrow b \in \mathrm{C}(a \rightarrow(a \rightarrow b))$;

(8) $\mathrm{C}(a \rightarrow b)=\mathrm{C}(\neg b \rightarrow \neg a)$;

(9) $E(\mathbb{L})$ contains all instances of all the theorems of $\mathbf{R}$, that is, for any $\varphi \in F m$, if $\vdash_{\mathbf{R}} \varphi$ then for any interpretation $\bar{a}$ on $\mathfrak{A}, \varphi^{\mathfrak{A}}(\bar{a}) \in E(\mathbb{L})$; and

(10) $\mathbb{L}$ is a model of $\mathbf{W R}$, that is, for any $\Gamma \cup\{\varphi\} \subseteq F m$, if $\Gamma \vdash_{\mathbf{W R}} \varphi$ then for any interpretation $\bar{a}$ over $\mathfrak{A}, \varphi^{\mathfrak{A}}(\bar{a}) \in \mathrm{C}\left(\Gamma^{\mathfrak{A}}(\bar{a})\right)$.

Proof: (1) Since trivially $a * c \in \mathrm{C}(a * c)$, by Proposition 4.5$] 3$ we get $c \rightarrow(a *$ $c) \in \mathrm{C}(a)$ which by assumption implies that $c \rightarrow(a * c) \in \mathrm{C}(b)$, which by the same result gives $a * c \in \mathrm{C}(b * c)$. Similarly, from $c \rightarrow b \in \mathrm{C}(c \rightarrow b)$ we obtain $c \rightarrow a \in$ $\mathrm{C}(c \rightarrow b)$.

(2) By 4.5.1 we know that $\mathrm{C}(b)=\mathrm{C}(\neg \neg b)$, and applying part (1) twice to this equality we obtain $\mathrm{C}(a \rightarrow b)=\mathrm{C}(a \rightarrow \neg \neg b)=\mathrm{C}(\neg \neg(a \rightarrow \neg \neg b))$ which by definition of $*$ gives $\mathrm{C}(a \rightarrow b)=\mathrm{C}(\neg(a * \neg b))$.

(3) From $\mathrm{C}(a) \subseteq \mathrm{C}(b)$ it follows by 4.5.1 that $\mathrm{C}(\neg b) \subseteq \mathrm{C}(\neg a)$, and then (1) gives $\mathrm{C}(c \rightarrow \neg b) \subseteq \mathrm{C}(c \rightarrow \neg a)$ which by definition of $*$ and 4.5.1 again implies $\mathrm{C}(c * a)=\mathrm{C}(\neg(c \rightarrow \neg a)) \subseteq \mathrm{C}(\neg(c \rightarrow \neg b))=\mathrm{C}(c * b)$. On the other hand, from $a * \neg c \in \mathrm{C}(a * \neg c)$ by 4.5 .3 we obtain $\neg c \rightarrow(a * \neg c) \in \mathrm{C}(a) \subseteq \mathrm{C}(b)$ which implies $a * \neg c \in \mathrm{C}(b * \neg c)$, and this by 4.5.1 again gives $\neg(b * \neg c) \in \mathrm{C}(\neg(a * \neg c))$. Now using (2) we have that $\mathrm{C}(b \rightarrow c)=\mathrm{C}(\neg(b * \neg c)) \subseteq \mathrm{C}(\neg(a * \neg c))=\mathrm{C}(a \rightarrow c)$.

(4) Assume that $b \in \mathrm{C}(a)$; since 4.5. 3 implies that $a \in \mathrm{C}((a \rightarrow a) * a)$, we have $b \in \mathrm{C}((a \rightarrow a) * a)$ which by the same reasoning gives $a \rightarrow b \in \mathrm{C}(a \rightarrow a) \subseteq E(\mathbb{L})$. Conversely, assume that $a \rightarrow b \in E(\mathbb{L})$; since the logic is finitary, satisfies DM2, and $\mathrm{C}(\varnothing)=\varnothing$, the definition of $E(\mathbb{L})$ means that there are $a_{1}, \ldots, a_{n} \in A$ such that, putting $c=\left(a_{1} \rightarrow a_{1}\right) \wedge \ldots \wedge\left(a_{n} \rightarrow a_{n}\right)$ we have $a \rightarrow b \in \mathrm{C}(c)$. On the other hand, observe that if $d_{1}, d_{2} \in A$ are such that $d_{i} \in \mathrm{C}\left(d_{i} \rightarrow d_{i}\right)$ then also $d_{1} \wedge d_{2}$ has this property, since by Conjunction we have $d_{1} \wedge d_{2} \in \mathrm{C}\left(\left(d_{1} \rightarrow d_{1}\right) \wedge\left(d_{2} \rightarrow d_{2}\right)\right)$ and then by 4.5 .5 and (3) $d_{1} \wedge d_{2} \in \mathrm{C}\left(\left(\left(d_{1} \rightarrow d_{1}\right) \wedge\left(d_{2} \rightarrow d_{2}\right)\right) \rightarrow\left(d_{1} \wedge d_{2}\right)\right) \subseteq$ $\mathrm{C}\left(\left(d_{1} \wedge d_{2}\right) \rightarrow\left(d_{1} \wedge d_{2}\right)\right)$; an easy induction allows us to conclude that $d_{1} \wedge \ldots \wedge$ $d_{n} \in \mathrm{C}\left(\left(d_{1} \wedge \ldots \wedge d_{n}\right) \rightarrow\left(d_{1} \wedge \ldots \wedge d_{n}\right)\right)$ whenever $d_{i} \in \mathrm{C}\left(d_{i} \rightarrow d_{i}\right)$. But this is the case for $d_{i}=a_{i} \rightarrow a_{i}$ because by 4.5.1 and 4.5.5 $a_{i} \rightarrow a_{i} \in \mathrm{C}\left(\left(a_{i} \rightarrow a_{i}\right) \rightarrow\left(a_{i} \rightarrow a_{i}\right)\right)$, 
so we have $c \in \mathrm{C}(c \rightarrow c)$ and as a consequence $a \rightarrow b \in \mathrm{C}(c \rightarrow c)$; then by 4.5.3 and the definition of $*$ we obtain $b \in \mathrm{C}((c \rightarrow c) * a)=\mathrm{C}(\neg((c \rightarrow c) \rightarrow \neg a))$. Finally, using 4.5.5 and 4.5.1 we get that $b \in \mathrm{C}(\neg((c \rightarrow c) \rightarrow \neg a)) \subseteq \mathrm{C}(\neg \neg a)=\mathrm{C}(a)$.

(5) From $a * b \in \mathrm{C}(a * b)$ and using $4.5,3$ and (4) we obtain $a \rightarrow(b \rightarrow(a * b)) \in$ $E(\mathbb{L})$ which by 4.5 .4 gives $b \rightarrow(a \rightarrow(a * b)) \in E(\mathbb{L})$; the same trick again now gives $a * b \in \mathrm{C}(b * a)$, which implies $\mathrm{C}(a * b)=\mathrm{C}(b * a)$.

(6) By definition $(b \rightarrow c) \rightarrow(b \rightarrow c) \in E(\mathbb{L})$, so by $4.5 .4 b \rightarrow((b \rightarrow c) \rightarrow c) \in$ $E(\mathbb{L})$ and by (4) $(b \rightarrow c) \rightarrow c \in \mathrm{C}(b)$; now we can use (1) to obtain $a \rightarrow((b \rightarrow c) \rightarrow$ $c) \in \mathrm{C}(a \rightarrow b)$ which by 4.5.4 again gives $(b \rightarrow c) \rightarrow(a \rightarrow c) \in \mathrm{C}(a \rightarrow b)$.

(7) Since by 4.51 we have that $\mathrm{C}(a)=\mathrm{C}(\neg \neg a)$ we can use (3), (2), (5), and the definition of $*$ to prove the equalities: $\mathrm{C}(a \rightarrow b)=\mathrm{C}(\neg \neg a \rightarrow b)=\mathrm{C}(\neg(\neg \neg a *$ $\neg b))=\mathrm{C}(\neg(\neg b * \neg \neg a))=\mathrm{C}(\neg b \rightarrow \neg a)$.

(8) From $a \rightarrow(a \rightarrow b) \in \mathrm{C}(a \rightarrow(a \rightarrow b))$ and (4) it follows that $(a \rightarrow(a \rightarrow$ $b)) \rightarrow(a \rightarrow(a \rightarrow b)) \in E(\mathbb{L})$ and then by 4.5.4, (3) and (4) we obtain first $a \rightarrow(a \rightarrow$ $((a \rightarrow(a \rightarrow b)) \rightarrow b)) \in E(\mathbb{L})$ and then $a \rightarrow((a \rightarrow(a \rightarrow b)) \rightarrow b) \in \mathrm{C}(a)$. Now by 4.5.2 this implies that $(a \rightarrow(a \rightarrow b)) \rightarrow b \in \mathrm{C}(a)$ and this by 4.5.3 and (5) finally gives $a \rightarrow b \in \mathrm{C}(a \rightarrow(a \rightarrow b))$.

(9) It is routine checking, using 4.5 .3 and the above properties, that $E(\mathbb{L})$ contains all instances of axioms of $\mathbf{R}$. Moreover, since $E(\mathbb{L}) \in C$, it is closed under the rules of Adjunction and Modus Ponens, by 4.5.1 and 4.5.2, and as a consequence $E(\mathbb{L})$ contains all instances of theorems of $\mathbf{R}$.

(10) If $\Gamma \vdash_{\mathbf{W R}} \varphi$ then by the finitarity and lack of theorems of $\vdash_{\mathbf{W R}}$ there are $\gamma_{1}, \ldots, \gamma_{n} \in \Gamma$ such that $\left\{\gamma_{1}, \ldots, \gamma_{n}\right\} \vdash_{\mathbf{W R}} \varphi$, that is, by Definition 3.1.3, that $\vdash_{\mathbf{R}}$ $\left(\gamma_{1} \wedge \ldots \wedge \gamma_{n}\right) \rightarrow \varphi$. By (9) this implies that for any interpretation $\bar{a}$ over $\mathfrak{A},\left(\gamma_{1}^{\mathfrak{A}}(\bar{a})\right.$ $\left.\wedge \ldots \wedge \gamma_{n}^{\mathfrak{A}}(\bar{a})\right) \rightarrow \varphi^{\mathfrak{A}}(\bar{a}) \in E(\mathbb{L})$. Now by (4) this is equivalent to saying that $\varphi^{\mathfrak{A}}(\bar{a}) \in \mathrm{C}\left(\gamma_{1}^{\mathfrak{A}}(\bar{a}) \wedge \ldots \wedge \gamma_{n}^{\mathfrak{A}}(\bar{a})\right)$ and by 4.51 this implies $\varphi^{\mathfrak{A}_{(}}(\bar{a}) \in \mathrm{C}\left(\Gamma^{\mathfrak{A}}(\bar{a})\right)$.

\section{Theorem 4.9 $\mathcal{S}_{\mathrm{WR}}=\mathrm{WR}$.}

Proof: By its own definition WR is finitary and does not have theorems. Now we are going to prove that $\mathbf{W R}$ is a model of $\mathcal{G}_{\mathbf{W R}}$, that is, to prove that it satisfies all the conditions in Proposition 4.5. First, we prove that $\langle\langle F m, \wedge, \neg\rangle, \vdash \mathbf{W R}\rangle$ is a De Morgan logic. It obviously satisfies DM1. From Lemma 3.2 and axioms R5 and R6 it follows that $\varphi, \psi \dashv \vdash_{\mathbf{W R}} \varphi \wedge \psi$, that is, that it satisfies DM2. Using axiom R10 and theorem R28 we see that $\gamma, \varphi \vdash_{\mathbf{W R}} \eta$ and $\gamma, \psi \vdash_{\mathbf{W R}} \eta$ imply $\gamma, \varphi \vee \psi \vdash_{\mathbf{W R}} \eta$; this, together with DM2 and axioms R8 and R9, implies that it satisfies DM3. Axiom $\mathrm{R} 13$ and its converse, which appear jointly as R20, immediately prove that it satisfies DM4, and R12 with Modus Ponens means that it satisfies DM5. Thus we have proved that WR satisfies 4.5.1. We have already noted that it is also closed under Modus Ponens (Lemma 3.2], that is, it satisfies 4.5.2. Theorem R30 gives 4.5]3, theorem R23 gives 4.5.4, and from RR Condition 4.5.5 immediately follows. Therefore, WR $\in$ $\mathbb{W R}(\mathfrak{F m})$ and by Theorem $4.7 \mathcal{S}_{\mathbf{W R}} \leq \mathbf{W R}$. There we have also proved that $\mathcal{S}_{\mathbf{W R}} \in$ $\mathbb{W} \mathbb{R}(\mathfrak{F m})$ and applying Proposition 4.810 to it we conclude that $\mathbf{W R} \leq \mathcal{S}_{\mathbf{W R}}$. As a consequence, $\mathbf{W R}=\mathcal{S}_{\mathbf{W R}}$.

Now that we have proved that $S_{\mathbf{W R}}$ equals $\mathbf{W R}$ we can regard the Gentzen calculus $\mathcal{G}_{\mathbf{W R}}$ merely as one of the presentations of the deductive system WR. Our aim 
is to show that in some sense it is not just one of but indeed it is the presentation, and we will do it by means of the notion of WR-logic; at present it is just a distinguished kind of model of a (Gentzen-style) presentation of $\mathbf{W R}$, but we will show that it is intrinsically associated with WR. More precisely, we will show that this notion equals that of full model of WR in the sense of [16, see below. The proof is based upon the so-called Bilogical Theorem 4.12, which will also establish an intrinsic relationship between WR and the class R. We need several definitions and elementary properties of abstract logics which we will now summarize; see 77 and 16$]$ for further details. To begin with, if $\mathbb{L}=\langle A, \mathrm{C}\rangle$ and $\mathbb{L}^{\prime}=\left\langle\mathfrak{A}^{\prime}, \mathrm{C}^{\prime}\right\rangle$ are two abstract logics of the same similarity type, we say that $h \in \operatorname{Hom}\left(\mathfrak{A}, \mathfrak{A}^{\prime}\right)$ is a logical morphism from $\mathbb{L}$ to $\mathbb{L}^{\prime}$ when it is continuous in the topological sense, that is, when for any $F \in C^{\prime}, h^{-1}(F) \in C$; if moreover $h$ is an epimorphism and it projectively generates $\mathbb{L}$ from $\mathbb{L}^{\prime}$ (which in this context means that $C=\left\{h^{-1}(F): F \in C^{\prime}\right\}$ ) then we say that $h$ is a bilogical morphism from $\mathbb{L}$ to $\mathbb{L}^{\prime}$. As a consequence, every bilogical morphism $h$ satisfies that $\mathrm{C}=h^{-1} \circ \mathrm{C}^{\prime} \circ h$ and that $\mathrm{C}^{\prime}=h \circ \mathrm{C} \circ h^{-1}$, as well as $F \in C \Longleftrightarrow h(F) \in C^{\prime}$ and $F \in C \Longrightarrow h^{-1}(h(F))=F$. We have:

Lemma 4.10 The property of being a WR-logic is preserved under bilogical morphisms, that is, if there is a bilogical morphism between two abstract logics (of suitable type) then one of them is a WR-logic if and only if the other one is.

Proof: $\quad$ Let $h$ be a bilogical morphism from $\mathbb{L}=\langle\mathfrak{A}, \mathrm{C}\rangle$ to $\mathbb{L}^{\prime}=\left\langle\mathfrak{A}^{\prime}, \mathrm{C}^{\prime}\right\rangle$; we are going to prove that $\mathbb{L} \in \mathbb{W} \mathbb{R}(\mathfrak{A})$ if and only if $\mathbb{L}^{\prime} \in \mathbb{W} \mathbb{R}\left(\mathfrak{A}^{\prime}\right)$ by seeing that one of them satisfies each of the conditions in Proposition 4.5 if and only if the other one satisfies it. First of all, observe that $h$ is also a bilogical morphism from the abstract logic $\langle\langle A, \wedge, \neg\rangle, \mathrm{C}\rangle$ to the abstract logic $\left\langle\left\langle A^{\prime}, \wedge, \neg\right\rangle, \mathrm{C}^{\prime}\right\rangle$ and then Proposition 3 of [18] implies that one of them is a De Morgan logic if and only if the other one is; moreover, it is trivial that $\mathrm{C}(\varnothing)=\varnothing$ if and only if $\mathrm{C}^{\prime}(\varnothing)=\varnothing$, thus we see that $\mathbb{L}$ satisfies 4.5. 1 if and only if $\mathbb{L}^{\prime}$ satisfies it. To show that the same holds for the remaining four conditions of Proposition 4.5 it is enough to observe that for any $a, b \in A$ it holds $b \in \mathrm{C}(a)$ if and only if it holds $h(b) \in \mathrm{C}^{\prime}(h(a))$, and take into account the fact that any bilogical morphism is onto; a direct inspection of 4.5 finishes the proof.

There are two equivalence relations naturally associated with every abstract $\operatorname{logic} \mathbb{L}=\langle\mathfrak{A}, \mathrm{C}\rangle$, introduced in [15] and [16]. The first one is called the Frege relation, and it is the abstract counterpart of the interderivability relation in a deductive system:

$$
\begin{aligned}
\Lambda(\mathbb{L}) & =\{\langle a, b\rangle \in A \times A: \mathrm{C}(a)=\mathrm{C}(b)\} \\
& =\{\langle a, b\rangle \in A \times A: \forall F \in C, a \in F \Longleftrightarrow b \in F\} .
\end{aligned}
$$

This relation leads us to consider the so-called logical congruences of $\mathbb{L}$, or congruences compatible with $\mathbb{L}$, defined as: Con $\mathbb{L}=\{\theta \in \operatorname{Con} \mathfrak{A}: \theta \subseteq \Lambda(\mathbb{L})\}$, that is, the congruences of the algebra that do not identify two elements unless they are interderivable. Every $\theta \in \operatorname{Con} \mathbb{L}$ determines a quotient logic $\mathbb{L} / \theta=\langle\mathfrak{A} / \theta, \mathrm{C} / \theta\rangle$ by defining $C / \theta=\{F / \theta: F \in C\}$, and then the canonical projection becomes a bilogical morphism (and conversely every bilogical morphism is determined by some logical congruence...). The second equivalence relation naturally associated with $\mathbb{L}$ is the 
so called Tarski congruence $\widetilde{\Omega}(\mathbb{L})=\max \operatorname{Con} \mathbb{L}$ (it is easy to see that such a maximum always exists). An abstract logic $\mathbb{L}$ is called reduced when $\widetilde{\Omega}(\mathbb{L})=\Delta_{A}$, that is, when the identity is the only logical congruence of $\mathbb{L}$. Every abstract logic can be reduced by factoring it by its Tarski congruence; in this way we obtain the reduction of $\mathbb{L}$, which is denoted by $\mathbb{L}^{*}=\left\langle\mathfrak{A}^{*}, \mathrm{C}^{*}\right\rangle$ where $\mathfrak{A}^{*}=\mathfrak{A} / \widetilde{\Omega}(\mathbb{L})$ and $C^{*}=C / \widetilde{\Omega}(\mathbb{L})$. Note that this reduction process can be applied only once, that is, we will identify $\mathfrak{A}^{* *}$ with $\mathfrak{A}^{*}$, and $\mathbb{L}^{* *}$ with $\mathbb{L}^{*}$, since they are isomorphic, because $\widetilde{\Omega}\left(\mathbb{L}^{*}\right)=\Delta_{A^{*}}$. On the other hand, we say that an abstract logic $\mathbb{L}$ satisfies the congruence property when $\widetilde{\Omega}(\mathbb{L})=\Lambda(\mathbb{L})$, that is, when $\Lambda(\mathbb{L}) \in$ Con $\mathfrak{A}$. A deductive system is called selfextensional in 27 when as an abstract logic it has the congruence property, that is, when the interderivability relation is a congruence of the formula algebra. We thus have:

Lemma 4.11 If $\mathbb{L} \in \mathbb{W} \mathbb{R}(\mathfrak{A})$ then $\Lambda(\mathbb{L})=\widetilde{\Omega}(\mathbb{L})$, that is, all the WR-logics satisfy the property of congruence; in particular the deductive system $\mathbf{W R}$ is selfextensional.

Proof: $\quad$ Let $\mathbb{L}=\langle\mathfrak{A}, \mathrm{C}\rangle \in \mathbb{W} \mathbb{R}(\mathfrak{A})$. By 4.5.1 the abstract logic $\langle\langle A, \wedge, \neg\rangle, \mathrm{C}\rangle$ is a De Morgan logic, and by [18], Theorem 3 we know that the Frege relation (denoted in that paper by $\theta(\mathbf{C})) \Lambda(\mathbb{L})$ is a congruence with respect to $\wedge \mathrm{i} \neg$. On the other hand, from the second parts of Proposition 4.8.1 and 4.8$] 3$ it follows that $\Lambda(\mathbb{L})$ is a congruence with respect to $\rightarrow$, therefore $\Lambda(\mathbb{L}) \in$ Con $\mathfrak{A}$. This implies that $\Lambda(\mathbb{L}) \in \operatorname{Con} \mathbb{L}$, and since in general $\widetilde{\Omega}(\mathbb{L}) \subseteq \Lambda(\mathbb{L})$ and $\widetilde{\Omega}(\mathbb{L})=\max \operatorname{Con}(\mathbb{L})$, we have $\Lambda(\mathbb{L})=$ $\widetilde{\Omega}(\mathbb{L})$.

Theorem 4.12 (Bilogical Theorem) Let $\mathbb{L}=\langle\mathfrak{A}, \mathcal{C}\rangle$ be an abstract logic of type $(2,2,1)$. Then the following conditions are equivalent:

(1) $\mathbb{L} \in \mathbb{W} \mathbb{R}(\mathfrak{A})$;

(2) $\mathfrak{A}^{*} \in \mathrm{R}$ and $C^{*}=\mathcal{F}_{\text {llt }} \mathfrak{A}^{*}$; and

(3) There is a bilogical morphism from $\mathbb{L}$ to a logic $\mathbb{L}_{0}=\left\langle\mathfrak{A}_{0}, \mathcal{C}_{0}\right\rangle$ where $\mathfrak{A}_{0} \in \mathrm{R}$ and $\mathcal{C}_{0}=\mathcal{F}_{\text {llt }} \mathfrak{A}_{0}$.

Proof: $\quad(1 \Rightarrow 2)$ : Since $\mathrm{R}$ is a variety we will prove that $\mathfrak{A}^{*} \in \mathrm{R}$ by showing that any equation true in $R$ is also true in $\mathfrak{A}^{*}$ : By Theorem 2.4.4 we have that $\varphi \approx \psi$ is true in $\mathbf{R}$ if and only if $\vdash_{\mathbf{R}} \varphi \leftrightarrow \psi$ and by the definition of $\mathbf{W R}$ this in turn is equivalent to $\varphi \dashv \vdash_{\mathbf{W R}} \psi$. Now if $\mathbb{L} \in \mathbb{W} \mathbb{R}(\mathfrak{A})$ then by Proposition 4.8.10 $\mathbb{L}$ is a model for WR which implies that $\left\langle\varphi^{\mathfrak{A}}(\bar{a}), \psi^{\mathfrak{A}}(\bar{a})\right\rangle \in \Lambda(\mathbb{L})$ for any interpretation $\bar{a}$ over $\mathfrak{A}$. By Lemma $4.11 \Lambda(\mathbb{L})=\widetilde{\Omega}(\mathbb{L})$, which implies that $\varphi^{\mathfrak{A}^{*}}(\pi \circ \bar{a})=\psi^{\mathfrak{A}^{*}}(\pi \circ \bar{a})$, where $\pi$ is the canonical projection from $\mathfrak{A}$ onto $\mathfrak{A}^{*}$; since $\pi$ is onto we conclude that $\varphi \approx \psi$ holds in $\mathfrak{A}^{*}$ and as a consequence that $\mathfrak{A}^{*} \in \mathrm{R}$. Finally from [18], Theorem 3 we obtain that $C^{*}=\mathcal{F}_{\text {llt }} \mathfrak{A}^{*} . \quad(2 \Rightarrow 3)$ is trivial since the canonical projection from $\mathfrak{A}$ onto $\mathfrak{A}^{*}$ is a bilogical morphism from $\mathbb{L}$ to $\mathbb{L}^{*}$. $(3 \Rightarrow 1)$ follows from Theorem 4.6 and Lemma 4.10 .

One of the consequences of this theorem is a characterization of the class $R$ of all $\mathbf{R}$-algebras in terms of the class of reduced WR-logics and conversely; this is contained in the following result, which can be regarded as a kind of abstract version of Theorem 3.9. in it we denote by $\mathbb{W} \mathbb{R}^{*}(\mathfrak{A})$ the class of all reduced WR-logics. 
Corollary 4.13 If $\mathbb{L}=\langle\mathfrak{A}, C\rangle$ is any abstract logic of type $(2,2,1)$, then $\mathbb{L} \in \mathbb{W} \mathbb{R}^{*}(\mathfrak{A})$ if and only if $\mathfrak{A} \in \mathrm{R}$ and $\mathcal{C}=\mathcal{F}_{\text {llt }} \mathfrak{A}$.

Proof: If $\mathbb{L}=\langle\mathfrak{A}, C\rangle \in \mathbb{W} \mathbb{R}^{*}(\mathfrak{A})$ then by Theorem 4.12 $\mathfrak{A}=\mathfrak{A}^{*} \in \mathrm{R}$ and $C=$ $C^{*}=\mathcal{F}_{\text {llt }} \mathfrak{A}^{*}=\mathcal{F}_{\text {llt }} \mathfrak{A}$. Conversely, if $\mathfrak{A} \in \mathrm{R}$ then by Theorem $4.6\left\langle\mathfrak{A}, \mathcal{F}_{\text {llt }} \mathfrak{A}\right\rangle \in$ $\mathbb{W} \mathbb{R}(\mathfrak{A})$ and then we can apply Lemma 4.11 which tells us that $\widetilde{\Omega}\left(\left\langle\mathfrak{A}, \mathcal{F}_{l} l t \mathfrak{A}\right\rangle\right)=$ $\Lambda\left(\left\langle\mathfrak{A}, \mathcal{F}_{\text {ll } l t} \mathfrak{A}\right\rangle\right)$, but this is the identity in every lattice, thus indeed $\left\langle\mathfrak{A}, \mathcal{F}_{\text {llt }} \mathfrak{A}\right\rangle \in$ $\mathbb{W} \mathbb{R}^{*}(\mathfrak{A})$.

Now we are prepared to prove that the class of WR-logics is intrinsically associated with the deductive system WR in the sense of [16, and at the same time we will prove a similar thing for the class $R$ of $\mathbf{R}$-algebras: in [16] the class of $\mathcal{S}$-algebras $\operatorname{Alg} S$ is introduced for every deductive system $\mathcal{S}$, and several results confirm that this is always a class of algebras naturally associated with $\mathcal{S}$. In our case $\operatorname{Alg} W \mathbf{R}=\{\mathfrak{A}$ : the abstract logic $\left\langle\mathfrak{A}, \mathcal{F}^{i} \mathbf{W} \mathbf{R}^{\mathfrak{A}\rangle}\right\rangle$ is reduced $\}$. On the other hand, we can associate a class of algebras with every Gentzen calculus by using the notion of model and the notion of reduced abstract logic; in our case, we say that an algebra $\mathfrak{A}=\langle A, \wedge, \rightarrow, \neg\rangle$ of type $(2,2,1)$ is a $\mathcal{G}_{\mathbf{W R}}$-algebra when $\mathfrak{A}$ is the algebra reduct of a reduced model of $\mathcal{G}_{\mathbf{W R}}$, that is, when there is an abstract logic $\mathbb{L}=\langle\mathfrak{A}, \mathrm{C}\rangle$ over $\mathfrak{A}$ such that $\mathbb{L}$ is a model of $\mathcal{G}_{\mathbf{W R}}$ and $\widetilde{\Omega}(\mathbb{L})=\Delta_{A}$; we denote by $\operatorname{Alg} \mathcal{G}_{\mathbf{W R}}$ the class of all $\mathcal{G}_{\mathbf{W R}}$-algebras. Finally in 16 another class of abstract logics is associated with every deductive system $\mathcal{S}$, the class of full models of $\mathcal{S}$; in our case, for every $\mathfrak{A}$ of type $(2,2,1)$ the class of full models of $\mathbf{W R}$ is the class $\mathcal{F M}$ od $\mathbf{W} \mathbf{R}^{\mathfrak{A}}=\left\{\mathbb{L}=\langle\mathfrak{A}, C\rangle: C^{*}=\mathcal{F} i_{\mathbf{W}} \mathbf{R}^{\mathfrak{A}^{*}}\right\}$. We then have:

\section{Theorem 4.14 $\operatorname{Alg} \mathcal{G}_{\mathbf{W R}}=\operatorname{AlgWR}=\mathrm{R}$ and $\mathbb{W} \mathbb{R}(\mathfrak{A})=\mathcal{F M o d}_{\mathbf{W R}} \mathfrak{A}$}

Proof: In Theorem 3.8 we have proved that RAlgWR $=\mathrm{R}$. In 16, Proposition 3.14 it is proved that for any deductive system $\mathcal{S}$, RAlg $S \subseteq \operatorname{Alg} S$ and that these classes of algebras generate the same variety; since in this case the smaller one is already a variety, they have to be equal, so $A \lg \mathbf{R}=R A \lg \mathbf{W}=R$. If $\mathfrak{A} \in R$ then by Corollary 4.13 $\left.\mathfrak{A}, \mathcal{F}_{l} l t \mathfrak{A}\right\rangle \in \mathbb{W}^{*}(\mathfrak{A})$, in particular this is a reduced model of $\mathcal{G}_{\mathbf{W R}}$, thus $\mathfrak{A} \in \operatorname{Alg} \mathcal{G}_{\mathbf{W R}}$. Conversely, if $\mathfrak{A} \in \operatorname{Alg} \mathcal{G}_{\mathbf{W R}}$ then there is some closure operator $\mathrm{C}$ over $\mathfrak{A}$ such that $\langle\mathfrak{A}, \mathrm{C}\rangle$ is a reduced model of $\mathcal{G}_{\mathbf{W R}}$. It is easy to see that then the closure operator $\overline{\mathrm{C}}$ defined over $\mathfrak{A}$ by: $\overline{\mathrm{C}}(\varnothing)=\varnothing, \overline{\mathrm{C}}(X)=\bigcup\{\mathrm{C}(F): F \subseteq X$ and $F$ finite is also a reduced model of $\mathcal{G}_{\mathbf{W R}}$, and it is finitary; according to Definition 4.4 it follows that $\langle\mathfrak{A}, \overline{\mathbf{C}}\rangle \in \mathbb{W} \mathbb{R}^{*}(\mathfrak{A})$, which again by Corollary 4.13 implies that $\mathfrak{A} \in \mathbf{R}$. Thus we have proved that $\operatorname{Alg} \mathcal{G}_{\mathbf{W R}}=\operatorname{Alg} \mathbf{W} \mathbf{R}=\mathrm{R}$. Now let $\mathbb{L} \in \mathbb{W} \mathbb{R}(\mathfrak{A})$; by Theorem 4.12 ve know that $\mathcal{C}^{*}=\mathcal{F}_{\text {llt }} \mathfrak{A}^{*}$ and that $\mathfrak{A}^{*} \in \mathrm{R}$, but in this

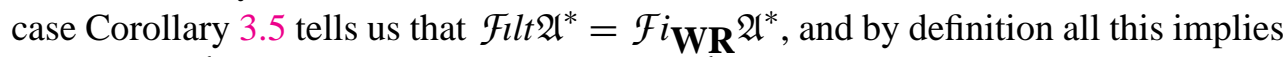
that $\mathbb{L} \in \mathcal{F M o d} \mathbf{W} \mathbf{R}^{\mathfrak{A}}$. Conversely, if $\mathbb{L} \in \mathcal{F} \mathcal{M}$ od $\mathbf{W} \mathbf{R}^{\mathfrak{A}}$ then it is easy to see, from the very definitions, that $\mathfrak{A}^{*} \in \operatorname{Alg} \mathbf{W R}$, that is, as we have proved, that $\mathfrak{A}^{*} \in R$, and we also obtain $C^{*}=\mathcal{F}_{i} \mathbf{W R} \mathfrak{A}^{*}=\mathcal{F}_{\text {llt }} \mathfrak{A}^{*}$; but then by Theorem $4.12\left\langle\left\{\mathfrak{A}^{*}, C^{*}\right\rangle \in \mathbb{W} \mathbb{R}(\mathfrak{A})\right.$, and this property is preserved under bilogical morphisms by Lemma 4.10, so also $\mathbb{L}=\langle\mathfrak{A}, C\rangle \in \mathbb{W} \mathbb{R}(\mathfrak{A})$. Thus exactly $\mathbb{W} \mathbb{R}(\mathfrak{A})=\mathcal{F} \mathcal{M}$ od $\mathbf{W} \mathbf{R}^{\mathfrak{A}}$.

Having identified the classes of algebras and of abstract logics associated with WR following the general scheme developed in 16 for any deductive system, we can obtain several consequences of some of the general results of this paper; let us record 
here that from the results of this section it follows that the deductive system WR is strongly self-extensional (all its full models have the congruence property), that the Gentzen calculus $\mathcal{G}_{\mathbf{W R}}$ is strongly adequate for it (the full models of WR are exactly the finitary models of $\mathcal{G}_{\mathbf{W R}}$ without theorems), and that this property uniquely determines $\mathcal{G}_{\mathbf{W R}}$ with respect to the deductive system $\mathbf{W R}$ (see more details in [16]).

\section{Corollary 4.15}

(1) For any algebra $\mathfrak{A}$ of type $(2,2,1)$, the ordered set $\langle\mathbb{W} \mathbb{R}(\mathfrak{A}), \leq\rangle$ is a complete lattice isomorphic to the lattice $\left\langle\operatorname{Con}_{\mathrm{R}}(\mathfrak{A}), \subseteq\right\rangle$, and the isomorphism is given by the mapping corresponding to the Tarski congruence: $\mathbb{L} \mapsto \widetilde{\Omega}(\mathbb{L})$.

(2) The algebraic category of $\mathbf{R}$-algebras (that is, the one with $\mathbf{R}$-algebras as objects and homomorphisms as arrows) is isomorphic to the category whose objects are the reduced WR-logics and whose arrows are all the logical morphisms between them.

(3) The second category of (2) is a full reflective subcategory of the category of all WR-logics with all logical morphisms.

Proof: (1) is a particular case of Theorems 2.28 and 2.29 of [16], which are completely general. (2) is a particular case of Theorem 2.33 of the same paper, which is also general. Finally (3) is a particular case of Theorem 2.42 of the same paper, which holds for strongly self-extensional deductive systems.

These results reinforce our claim that the classes of $\mathbf{R}$-algebras and of WR-logics are intrinsically associated with the deductive system WR.

5 The R-logics In this section we are going to associate a class of abstract logics with the deductive system $\mathbf{R}$ in a natural way. However, we do not have a Gentzen calculus which defines $\mathbf{R}$ in a similar way as we had in the preceding section for WR, and thus we cannot start with its models. Instead we take the abstract counterpart of Theorem 3.3. which tells us that the theories of $\mathbf{R}$ are the theories of $\mathbf{W R}$ containing the formulas of the form $\varphi \rightarrow \varphi$, that is, the theories of WR containing the theory generated by these formulas, which we have designated by $E(\mathbf{W R})$. The procedure uses the notion of axiomatic extension: For any abstract logic $\mathbb{L}=\langle\mathfrak{A}, \mathrm{C}\rangle$ and any $X \subseteq A$ the axiomatic extension of $\mathbb{L}$ by $X$ is the abstract logic $\mathbb{L}^{X}=\left\langle\mathfrak{A}, \mathrm{C}^{X}\right\rangle$ where $\mathrm{C}^{X}$ is the closure operator corresponding to the closure system $C^{X}=\{T \in C: X \subseteq T\}$. We then put:

Definition 5.1 Let $\mathbb{L}=\langle\mathfrak{A}, \mathrm{C}\rangle$ be an abstract logic of type $(2,2,1)$. We say that $\mathbb{L}$ is an $\mathbf{R}$-logic when there is a closure operator $\mathrm{C}^{\prime}$ over $A$ such that the abstract logic $\mathbb{L}^{\prime}=\left\langle\mathfrak{A}, \mathbf{C}^{\prime}\right\rangle$ is a $\mathbf{W R}$-logic and $\mathbb{L}$ is the axiomatic extension of $\mathbb{L}^{\prime}$ by $E\left(\mathbb{L}^{\prime}\right)$. For every algebra $\mathfrak{A}$ we will denote by $\mathbb{R}(\mathfrak{A})$ the set of all R-logics defined over $\mathfrak{A}$.

We shall see at once that the logic $\mathbb{L}^{\prime}$ is unique, whenever it exists, so that the definition makes full sense. Initially the connection of these $\mathbf{R}$-logics with the deductive system $\mathbf{R}$ is rather indirect; the object of this last section is to prove that they are the abstract logics naturally associated with $\mathbf{R}$ by the procedure of [16, and to such end we will prove the corresponding Bilogical Theorem.

The following result establishes the uniqueness of the associated WR-logic used in Definition 5.1. and thus eliminates any ambiguity in it. Actually it says more: it 
says that the relationship between any $\mathbf{R}$-logic and its associated $\mathbf{W R}$-logic is the abstract version of the procedure we used to define the deductive system WR starting from the deductive system $\mathbf{R}$. As a consequence we find a bijective correspondence between $\mathbb{W} \mathbb{R}(\mathfrak{A})$ and $\mathbb{R}(\mathfrak{A})$.

Theorem 5.2 If $\mathbb{L}=\langle\mathfrak{A}, \mathrm{C}\rangle$ is an $\mathbf{R}$-logic and $\mathbb{L}^{\prime}=\left\langle\mathfrak{A}, \mathrm{C}^{\prime}\right\rangle$ is any WR-logic satisfying the conditions of Definition 5.1. then for any $X \subseteq A$ it holds that

$$
\mathrm{C}^{\prime}(X)=\left\{a \in A: \exists a_{1}, \ldots, a_{n} \in X \text { with }\left(a_{1} \wedge \ldots \wedge a_{n}\right) \rightarrow a \in \mathrm{C}(\varnothing)\right\}
$$

and as a consequence this $\mathbb{L}^{\prime}$ is uniquely determined.

Proof: By definition $\mathbb{L}^{\prime}$ satisfies $\mathrm{C}^{\prime}(\varnothing)=\varnothing$, which implies that the above condition is fulfilled for $X=\varnothing$. Suppose that $X \neq \varnothing$. Since also by definition $\mathbb{L}^{\prime}$ is finitary and satisfies DM2, $a \in \mathrm{C}^{\prime}(X)$ if and only if there are $a_{1}, \ldots, a_{n} \in X$ with $a \in$ $\mathrm{C}^{\prime}\left(a_{1}, \wedge \ldots \wedge, a_{n}\right)$ which by Proposition 4.8.4 is equivalent to $\left(a_{1} \wedge \ldots \wedge a_{n}\right) \rightarrow a \in$ $E\left(\mathbb{L}^{\prime}\right)$. But $\mathrm{C}(\varnothing)=\mathrm{C}^{\prime} E\left(\mathbb{L}^{\prime}\right)(\varnothing)=\mathrm{C}^{\prime}\left(E\left(\mathbb{L}^{\prime}\right)\right)=E\left(\mathbb{L}^{\prime}\right)$, which establishes the desired characterization of $\mathrm{C}^{\prime}$ and therefore uniquely determines $\mathbb{L}^{\prime}$.

Corollary 5.3 For every algebra $\mathfrak{A}$ the ordered sets $\langle\mathbb{W} \mathbb{R}(\mathfrak{A}), \leq\rangle$ and $\langle\mathbb{R}(\mathfrak{A}), \leq\rangle$ are isomorphic.

Proof: Consider the mappings $\mathbb{L} \mapsto \mathbb{L}^{E(\mathbb{L})}$, which goes from $\mathbb{W} \mathbb{R}(\mathfrak{A})$ to $\mathbb{R}(\mathfrak{A})$, and $\mathbb{L} \mapsto \mathbb{L}^{\prime}$, which goes from $\mathbb{R}(\mathfrak{A})$ to $\mathbb{W} \mathbb{R}(\mathfrak{A})$. It has already been established that they are well-defined. The first one is order-preserving by the definition of axiomatic extension, and the second one is also so by Theorem 5.2. Definition 5.1 says that $\left(\mathbb{L}^{\prime}\right)^{E\left(\mathbb{L}^{\prime}\right)}=\mathbb{L}$. Finally, if $\mathbb{L} \in \mathbb{W} \mathbb{R}(\mathfrak{A})$ we see that $\left(\mathbb{L}^{E(\mathbb{L})}\right)^{\prime}=\mathbb{L}: a \in\left(\mathrm{C}^{E(\mathbb{L})}\right)^{\prime}(X)$ if and only if there are $a_{1}, \ldots, a_{n} \in X$ such that $\left(a_{1} \wedge \ldots \wedge a_{n}\right) \rightarrow a \in \mathrm{C}^{E(\mathbb{L})}(\varnothing)=$ $E(\mathbb{L})$ and by Proposition 4.8.4 this is equivalent to $a \in \mathrm{C}\left(a_{1} \wedge \ldots \wedge a_{n}\right)=\mathrm{C}\left(a_{1}, \ldots\right.$, $\left.a_{n}\right) \subseteq \mathrm{C}(X)$. Therefore these two mappings are monotone, bijective and inverse to one another, that is, they are the required order-isomorphisms.

Among the consequences of this order-isomorphism we have:

Proposition 5.4 $\quad \mathbf{R}=\min \mathbb{R}(\mathfrak{F m})$, and for every $\mathfrak{A} \in R,\langle\mathfrak{A}, \mathcal{D e d} \mathfrak{A}\rangle=\min \mathbb{R}(\mathfrak{A})$.

Proof: $\quad$ To prove that $\mathbf{R}=\min \mathbb{R}(\mathfrak{F m})$ it is enough to use Theorems 3.3, 4.7 and 4.9 (here the auxiliary $\mathbf{W R}$-logic $\mathbf{R}^{\prime}$ is precisely $\mathbf{W R}$ !). If $\mathfrak{A} \in R$ then by Theorem 4.6 we know that $\left\langle\mathfrak{A}, \mathcal{F}_{l} l t \mathfrak{A}\right\rangle=\min \mathbb{W} \mathbb{R}(\mathfrak{A})$; on the other hand by definition $E\left(\left\langle\mathfrak{A}, \mathcal{F}_{l} l t \mathfrak{A}\right\rangle\right)$ $=E(\mathfrak{A})$, thus $\operatorname{Ded} \mathfrak{A}=\left(\mathcal{F}_{l} l t \mathfrak{A}\right)^{E\left(\left\langle\mathfrak{A}, \mathcal{F}_{l} l t \mathfrak{A}\right\rangle\right)}$. From Corollary 5.3 t follows that $\langle\mathfrak{A}$, $\operatorname{Ded} \mathfrak{A}\rangle=\min \mathbb{R}(\mathfrak{A})$.

Let $\mathbb{L}=\langle\mathfrak{A}, \mathrm{C}\rangle \in \mathbb{R}(\mathfrak{A})$ and let $\mathbb{L}^{\prime}$ be its associated WR-logic. Then by the definitions we have $\varnothing \notin C$ while $\varnothing=\mathrm{C}^{\prime}(\varnothing) \in C^{\prime}$; as a consequence we always have $\mathbb{L} \neq \mathbb{L}^{\prime}$. However, we might suspect that this is the only difference between the two logics. Presenting this in a different way, we may ask under what conditions for every $a \in A, \mathrm{C}(a)=\mathrm{C}^{\prime}(a)$. This would amount to the coincidence between $\mathbb{L}^{\prime}$ and the greatest pseudoaxiomatic logic contained in $\mathbb{L}$, which is obtained by just adding $\varnothing$ to $C$; note that in Section 3 we asked (and answered in the negative) a similar question between the deductive systems $\mathbf{W R}$ and $\mathbf{R}$. It happens that in the general case the answer is related to the notion of classical logic. Following [6], p. 44, we say that an abstract logic $\mathbb{L}=\langle\mathfrak{A}, \mathbb{C}\rangle$, of type $(2,1)$ with $\mathfrak{A}=\langle A, \vee, \neg\rangle$, is classical when: 
(1) $\mathrm{C}$ is finitary;

(2) $\forall X \cup\{a, b\} \subseteq A, \mathrm{C}(X, a \vee b)=\mathrm{C}(X, a) \cap \mathrm{C}(X, b)$ (Disjunction);

(3) $\forall X \cup\{a\} \subseteq A, a \in \mathrm{C}(X) \Longleftrightarrow \mathrm{C}(X, \neg a)=A$ (Reductio ad Absurdum).

We then have:

Theorem 5.5 Let $\mathbb{L}=\langle\mathfrak{A}, C\rangle \in \mathbb{R}(\mathfrak{A})$ be an $\mathbf{R}$-logic, and let $\mathbb{L}^{\prime}=\left\langle\mathfrak{A}, C^{\prime}\right\rangle \in$ $\mathbb{W} \mathbb{R}(\mathfrak{A})$ be the unique $\mathbf{W R}$-logic associated with $\mathbb{L}$. Then it holds that $C^{\prime}=C \cup\{\varnothing\}$ if and only if the logic $\langle\langle A, \vee, \neg\rangle, C\rangle$ is a classical logic. Moreover in this case $\mathrm{C}(a \rightarrow b)=\mathrm{C}(\neg a \vee b)$ and $\mathrm{C}(a * b)=\mathrm{C}(a \wedge b)$.

Proof: $\quad(\Rightarrow)$ By assumption we have that for every $X \subseteq A$, if $X \neq \varnothing$ then $\mathrm{C}^{\prime}(X)=$ $\mathrm{C}(X)$. If $a \in E\left(\mathbb{L}^{\prime}\right)$ we have $\mathrm{C}^{\prime}(a)=\mathrm{C}(a)=\mathrm{C}^{\prime}\left(a, E\left(\mathbb{L}^{\prime}\right)\right)=\mathrm{C}^{\prime}\left(E\left(\mathbb{L}^{\prime}\right)\right)=E\left(\mathbb{L}^{\prime}\right)$, and therefore for all $a, b \in E\left(\mathbb{L}^{\prime}\right), \mathrm{C}^{\prime}(a)=\mathrm{C}^{\prime}(b)=E\left(\mathbb{L}^{\prime}\right)$ and thus $\langle a, b\rangle \in \Lambda\left(\mathbb{L}^{\prime}\right)=$ $\widetilde{\Omega}\left(\mathbb{L}^{\prime}\right)$, that is, $a^{*}=b^{*}$ and thus $E\left(\mathfrak{A}^{*}\right)$ has only one element. Note that by Theorem $4.12 \mathfrak{A}^{*} \in \mathrm{R}$. Then it is easy to see that this singleton is the maximum of the quotient set. On the other hand, by R53 we have that for any $a \in A, \neg a^{*} \vee a^{*} \in$ $E\left(\mathfrak{A}^{*}\right)$. As a consequence, the algebra $\left\langle A^{*}, \vee, \neg\right\rangle$ is a Boolean algebra. By Theorem $4.12\left(C^{\prime}\right)^{*}=\mathcal{F}_{\text {llt }} \mathfrak{A}^{*}$ which comprises all lattice filters of $\mathfrak{A}^{*}$ plus the empty set; since $\left(C^{\prime}\right)^{*}=C^{*} \cup\{\varnothing\}, C^{*}$ must be the family of all lattice filters of $\mathfrak{A}^{*}$, that is, $\left\langle\left\langle\mathfrak{A}^{*}, \vee, \neg\right\rangle, C^{*}\right\rangle$ is a Boolean logic, and this implies that $\langle\langle\mathfrak{A}, \vee, \neg\rangle, C\rangle$ is a classical logic (see [6], p. 44 and Theorem 3, for instance). Finally, to prove that $\mathrm{C}(a \rightarrow b)=\mathrm{C}(\neg a \vee b)$ we can use the properties stated in Proposition 4.8. because by assumption $\mathrm{C}$ and $\mathrm{C}^{\prime}$ agree on nonempty sets: since $\mathfrak{A}^{*}$ is a Boolean algebra and $E\left(\mathfrak{A}^{*}\right)=\{1\}$, for any $a, b \in A$ we have that $b^{*} \leq a^{*} \rightarrow a^{*}$, so $a \rightarrow a \in \mathrm{C}(b)$ and $a \in \mathrm{C}(a * b)$; from this and $\mathrm{R} 55$ it follows that $\mathrm{C}(a \wedge b)=\mathrm{C}(a, b)=\mathrm{C}(a * b)$ and $\mathrm{C}(a \rightarrow b)=\mathrm{C}(\neg(a * \neg b))=\mathrm{C}(\neg(a \wedge \neg b))=\mathrm{C}(\neg a \vee b)$.

$(\Leftarrow)$ If $\langle\langle A, \vee, \neg\rangle, C\rangle$ is classical, again by [6], Theorem 3 we know there is a bilogical morphism $h$ from $\langle\langle A, \vee, \neg\rangle, \mathrm{C}\rangle$ onto a Boolean logic $\left\langle\mathfrak{B}, \mathcal{F}_{l} l t^{o} \mathfrak{B}\right\rangle$ where $\mathcal{F}_{l} l t^{o} \mathfrak{B}$ is the family of all lattice filters of $\mathfrak{B}$; by adding $\varnothing$ to it we obtain $\mathcal{F}_{l} l t \mathfrak{B}$, but for all nonempty $X \subseteq B$, Filt ${ }^{o}(X)=\operatorname{Filt}(X)$, so Filt $(h(a \rightarrow b))=h(\mathrm{C}(a \rightarrow b))=$ $h(\mathrm{C}(\neg a \vee \neg b))=$ Filt $(h(\neg a \vee b))=$ Filt $(\neg h(a) \vee h(b))$. Therefore $h(a \rightarrow b)=$ $\neg h(a) \vee h(b)$ and thus $h$ is also a bilogical morphism from $\langle\mathfrak{A}, C \cup\{\varnothing\}\rangle$ onto the abstract logic which results from $\left\langle\mathfrak{B}, \mathcal{F}_{\text {llt }} \mathfrak{B}\right\rangle$ after changing $\mathfrak{B}$ by its $\langle\wedge, \rightarrow, \neg\rangle$-reduct, where $\rightarrow$ is material implication; it is easy to see that the resulting abstract logic is a WR-logic, so Lemma 4.10 tells us that $\langle\mathfrak{A}, C \cup\{\varnothing\}\rangle \in \mathbb{W} \mathbb{R}(\mathfrak{A})$ and by Theorem 5.2 this implies that $C \cup\{\varnothing\}=C^{\prime}$.

Now before proving the bilogical theorem for $\mathbb{R}(\mathfrak{A})$ we see that the property of being an $\mathbf{R}$-logic is preserved under bilogical morphisms.

Lemma 5.6 Let h be a bilogical morphism from $\mathbb{L}_{1}=\left\langle\mathfrak{A}_{1}, \mathrm{C}_{1}\right\rangle$ onto $\mathbb{L}_{2}=\left\langle\mathfrak{A}_{2}, \mathrm{C}_{2}\right\rangle$. Then $\mathbb{L}_{1}$ is an $\mathbf{R}$-logic if and only if $\mathbb{L}_{2}$ is an $\mathbf{R}$-logic.

Proof: Assuming that $\mathbb{L}_{1} \in \mathbb{R}\left(\mathfrak{A}_{1}\right)$ we prove that $\mathbb{L}_{2} \in \mathbb{R}\left(\mathfrak{A}_{2}\right)$. Since $\mathbb{L}_{1}^{\prime} \in \mathbb{W} \mathbb{R}\left(\mathfrak{A}_{1}\right)$, Lemma 4.10 implies that $h$ is a bilogical morphism from $\mathbb{L}_{1}^{\prime}$ onto the abstract logic $\mathbb{L}^{\prime}=\left\langle\mathfrak{A}_{2}, \mathrm{C}^{\prime}\right\rangle \in \mathbb{W} \mathbb{R}\left(\mathfrak{A}_{2}\right)$, where $\mathrm{C}^{\prime}=h \circ \mathrm{C}_{1}^{\prime} \circ h^{-1}$ and $E\left(\mathbb{L}^{\prime}\right)=h\left(E\left(\mathbb{L}_{1}^{\prime}\right)\right)$; then using that $h$ is a bilogical morphism from $\mathbb{L}_{1}$ onto $\mathbb{L}_{2}$ and that $\mathbb{L}_{1} \in \mathbb{R}\left(\mathfrak{A}_{1}\right)$, we obtain that for any $X \subseteq A_{2}, \mathrm{C}_{2}(X)=h\left(\mathrm{C}_{1}\left(h^{-1}(X)\right)=h\left(C_{1}^{\prime}\left(h^{-1}(X), E\left(\mathbb{L}_{1}^{\prime}\right)\right)\right)=\right.$ $\left(h \circ \mathrm{C}_{1}^{\prime} \circ h^{-1}\right)\left(X, h\left(E\left(\mathbb{L}_{1}^{\prime}\right)\right)=\mathrm{C}^{\prime}\left(X, E\left(\mathbb{L}^{\prime}\right)\right)\right.$. Since $\mathbb{L}^{\prime}$ is a WR-logic it follows from 
Definition 5.1 that $\mathbb{L}_{2} \in \mathbb{R}\left(\mathfrak{A}_{2}\right)$. A similar process shows that $\mathbb{L}_{2} \in \mathbb{R}\left(\mathfrak{A}_{2}\right)$ implies $\mathbb{L}_{1} \in \mathbb{R}\left(\mathfrak{A}_{1}\right)$.

Theorem 5.7 (Bilogical Theorem) If $\mathbb{L}=\langle\mathfrak{A}, \mathrm{C}\rangle$ is an abstract logic of type $(2,2,1)$ then the following conditions are equivalent:

(1) $\mathbb{L} \in \mathbb{R}(\mathfrak{A})$;

(2) $\mathfrak{A}^{*} \in \mathrm{R}$ and $C^{*}=\operatorname{Ded}^{*}$; and

(3) There is a bilogical morphism between $\mathbb{L}$ and an abstract logic $\left\langle\mathfrak{A}_{0}, \mathcal{C}_{0}\right\rangle$, where $\mathfrak{A}_{0} \in \mathrm{R}$ and $C_{0}=\mathcal{D e d}$.

Proof: $\quad(1 \Rightarrow 2)$ : We begin by proving that $\widetilde{\Omega}(\mathbb{L})=\widetilde{\Omega}\left(\mathbb{L}^{\prime}\right)$. By Lemma 4.11 $\widetilde{\Omega}\left(\mathbb{L}^{\prime}\right)=\Lambda\left(\mathbb{L}^{\prime}\right) \in$ Con $\mathfrak{A}$, and since $\langle a, b\rangle \in \Lambda\left(\mathbb{L}^{\prime}\right) \Longleftrightarrow \mathrm{C}^{\prime}(a)=\mathrm{C}^{\prime}(b) \Longrightarrow$ $\mathrm{C}(a)$ $\mathrm{C}^{\prime}\left(a, E\left(\mathbb{L}^{\prime}\right)\right)=\mathrm{C}^{\prime}\left(b, E\left(\mathbb{L}^{\prime}\right)\right)=\mathrm{C}(b)$ it follows that $\widetilde{\Omega}\left(\mathbb{L}^{\prime}\right) \in$ Con $\mathbb{L}$; now using Proposition 4.8.4 we have $\langle a, b\rangle \in \widetilde{\Omega}\left(\mathbb{L}^{\prime}\right) \Longleftrightarrow \mathrm{C}^{\prime}(a)=\mathrm{C}^{\prime}(b) \Longleftrightarrow a \leftrightarrow b \in$ $E\left(\mathbb{L}^{\prime}\right)=\mathrm{C}(\varnothing)$ and by [7], Theorem IX.6, this implies that $\widetilde{\Omega}\left(\mathbb{L}^{\prime}\right)=\widetilde{\Omega}(\mathbb{L})$. Thus we can write $\mathfrak{A}^{*}=\mathfrak{A} / \widetilde{\Omega}(\mathbb{L})=\mathfrak{A} / \widetilde{\Omega}\left(\mathbb{L}^{\prime}\right)$, and by Theorem $4.12 \mathfrak{A}^{*} \in \mathrm{R}$ and $\left(C^{\prime}\right)^{*}=$ $\mathcal{F}_{\text {flt }} \mathfrak{A}^{*}$. Finally, since $E\left(\mathbb{L}^{\prime}\right) / \widetilde{\Omega}(\mathbb{L})=E\left(\left(\mathbb{L}^{\prime}\right)^{*}\right)=E\left(\mathfrak{A}^{*}\right)$, we have $C^{*}=\{F / \widetilde{\Omega}(\mathbb{L})$ : $F \in C\}=\left\{F / \widetilde{\Omega}\left(\mathbb{L}^{\prime}\right): F \in C^{\prime}\right.$ with $\left.E\left(\mathbb{L}^{\prime}\right) \subseteq F\right\}=\left\{F \in \mathcal{F}_{l} l t \mathfrak{A}^{*}: E\left(\mathfrak{A}^{*}\right) \subseteq F\right\}=$ $\mathcal{D e d}^{*}$. $(2 \Rightarrow 3)$ : Just take $\mathfrak{A}_{0}=\mathfrak{A}^{*}$, and the projection will be the required bilogical morphism. $(3 \Rightarrow 1)$ : Just apply Proposition 5.4 and Lemma 5.6.

In the course of the above proof we have seen that an R-logic and its associated WR-logic have the same associated Tarski congruence; we will later make use of this fact. In Lemma 4.11 we proved that WR-logics have the congruence property, so we suspect that in general $\mathbf{R}$-logics do not have it. To confirm this it is enough to show that this is the case for the simplest $\mathbf{R}$-logic, namely the deductive system $\mathbf{R}$ itself. In 16 a deductive system is called quasi-Fregean when all its axiomatic extensions, as abstract logics, have the congruence property.

Proposition 5.8 The deductive system $\mathbf{R}$ is not self-extensional, and the deductive system WR is not quasi-Fregean.

Proof: Assume that $\mathbf{R}$ is self-extensional. Then for any $\varphi, \psi \in F m$ we would have that $\varphi \vdash_{\mathbf{R}} \psi \Rightarrow \varphi \rightarrow \varphi \vdash_{\mathbf{R}} \varphi \rightarrow \psi$ and conversely by modus ponens $\varphi \vdash_{\mathbf{R}} \psi \Longleftrightarrow$ $\varphi \rightarrow \varphi \vdash_{\mathbf{R}} \varphi \rightarrow \psi$. Thus, taking Definition 3.1.3 into account, we would obtain $\varphi \vdash_{\mathbf{R}} \psi \Longleftrightarrow \vdash_{\mathbf{R}} \varphi \rightarrow \psi \Longleftrightarrow \varphi \vdash_{\mathbf{W R}} \psi$, and this equivalence is not true as we showed after Theorem 3.3. This shows that $\mathbf{R}$ cannot be self-extensional. Since it is an axiomatic extension of $\mathbf{W R}$, as shown in Theorem 3.3. it follows that WR cannot be quasi-Fregean.

It is interesting, for theoretical reasons concerning the general approach contained in [16], to notice that WR is an example of a non-protoalgebraic and strongly selfextensional deductive system which is not quasi-Fregean.

Now the bilogical theorem gives a characterization of the class $\mathbb{R}^{*}(\mathfrak{A})$ of the reduced $\mathbf{R}$-logics over a given algebra $\mathfrak{A}=\langle A, \wedge, \rightarrow, \neg\rangle$ of type $(2,2,1)$, by means of the class of $\mathbf{R}$-algebras, in a form completely parallel to that of the case of WRlogics stated in Corollary 3.13. 
Corollary 5.9 Let $\mathbb{L}=\langle\mathfrak{A}, C\rangle$ be an abstract logic of type $(2,2,1)$. Then $\mathbb{L} \in \mathbb{R}^{*}(\mathfrak{A})$ if and only if $\mathfrak{A} \in \mathrm{R}$ and $C=\mathcal{D e d} \mathfrak{A}$.

Proof: If $\mathbb{L}=\langle\mathfrak{A}, C\rangle \in \mathbb{R}^{*}(\mathfrak{A})$ then by Theorem 5.7 $\mathfrak{A}=\mathfrak{A}^{*} \in \mathrm{R}$ and $C=$ $C^{*}=\operatorname{Ded}^{*} \mathfrak{A}^{*}=\operatorname{Ded} \mathfrak{A}$. Conversely, if $\mathfrak{A} \in \mathrm{R}$ then by Proposition 5.4 we have that $\langle\mathfrak{A}, \operatorname{Ded} \mathfrak{A}\rangle \in \mathbb{R}(\mathfrak{A})$ and it is reduced, because, as remarked after the proof of Theo$\operatorname{rem}$ 5.7 and using Lemma 4.11, $\widetilde{\Omega}(\langle\mathfrak{A}, \mathcal{D} e d \mathfrak{A}\rangle)=\widetilde{\Omega}\left(\left\langle\mathfrak{A}, \mathcal{F}_{l} l t \mathfrak{A}\right\rangle\right)=\Lambda\left(\left\langle\mathfrak{A}, \mathcal{F}_{l} l t \mathfrak{A}\right\rangle\right)=$ $\Delta_{A}$.

Now we can incorporate the general notions introduced in 16$]$ in a similar way as we did in the last part of Section 4; the difference is that here we do not use any Gentzen calculus associated with the deductive system $\mathbf{R}$ in order to characterize the class of abstract logics we obtain. In the case of $\mathbf{R}$, the class of algebras associated with it is $\operatorname{Alg} \mathbf{R}=\{\mathfrak{A}:\langle\mathfrak{A}, \mathcal{F} i \mathbf{R} \mathfrak{A}\rangle$ is reduced $\}$ and for every algebra $\mathfrak{A}$ of suitable type, the class of abstract logics over $\mathfrak{A}$ associated with $\mathbf{R}$, called its full models, is $\mathcal{F M o d}_{\mathbf{R}} \mathfrak{A}=\left\{\mathbb{L}=\langle\mathfrak{A}, C\rangle: C^{*}=\mathcal{F} i \mathbf{R}^{\left.\mathfrak{A}^{*}\right\}}\right.$. In [16] it is shown that these notions are intrinsically associated with $\mathbf{R}$ in a natural form. Therefore the following result is important:

Theorem 5.10 $\quad \operatorname{Alg} \mathbf{R}=\mathrm{R}$ and for every $\mathfrak{A}$ of type $(2,2,1), \mathcal{F M o d} \mathbf{R}^{\mathfrak{A}}=\mathbb{R}(\mathfrak{A})$.

Proof: In [16], Proposition 3.2 it is proved that for any protoalgebraic deductive system $\mathcal{S}, \operatorname{RAlg} S=\operatorname{Alg} S$; we already know that $\mathbf{R}$ is algebraizable, hence it is also protoalgebraic, and we proved in Theorem 2.6 hat $R A \lg \mathbf{R}=R$, therefore $A \lg \mathbf{R}=R$ as well. On the other hand, from the definitions of full models of $\mathcal{S}$ and of $\mathcal{S}$-algebra it follows in general that $\mathbb{L} \in \mathcal{F} \mathcal{M}$ d $_{S} \mathfrak{A}$ if and only if $\mathfrak{A}^{*} \in \operatorname{Alg} S$ and $C^{*}=\mathcal{F}_{\mathcal{S}} \mathfrak{A}^{*}$. Therefore, after having proved that $\operatorname{Alg} \mathbf{R}=\mathrm{R}$, our Theorems 5.7 and 2.4.2 establish the equality $\mathcal{F M o d} \mathbf{R}_{\mathbf{A}}=\mathbb{R}(\mathfrak{A})$ for every algebra $\mathfrak{A}$ of suitable type.

At this point several general results of 16 can be applied to the classes of $\mathbf{R}$ algebras and of $\mathbf{R}$-logics:

Corollary 5.11 For any algebra $\mathfrak{A}=\langle A, \wedge, \rightarrow, \neg\rangle$ of type $(2,2,1)$ the following hold:

(1) The mapping $F \longmapsto\left\langle\mathfrak{A},\left(\mathcal{F} i_{\mathbf{R}} \mathfrak{A}\right)^{F}\right\rangle$ is an isomorphism between the lattices $\mathcal{F}_{i} \mathbf{R}^{\mathfrak{A}}$ and $\mathcal{F M o d} \mathbf{R}_{\mathbf{R}} \mathfrak{A}$. Therefore, the $\mathbf{R}$-logics are the abstract logics whose associated closure system consists of all $\mathbf{R}$-filters containing a given $\mathbf{R}$-filter.

(2) The set $\langle\mathbb{R}(\mathfrak{A}), \leq\rangle$ is a complete lattice isomorphic to the lattice $\left\langle\operatorname{Con}_{\mathrm{R}}(\mathfrak{A}), \subseteq\right.$ \rangle ; the isomorphism is given by the Tarski operator $\widetilde{\Omega}$ on $\mathbb{R}(\mathfrak{A})$, and the first element of $\mathbb{R}(\mathfrak{A})$ is the abstract logic $\left\langle\mathfrak{A}, \mathcal{F} i \mathbf{R}_{\mathbf{A}} \mathfrak{A}\right\rangle$.

(3) The algebraic category determined by the class of $\mathbf{R}$-algebras (that is, the category whose objects are $\mathbf{R}$-algebras and whose arrows are homomorphisms) is isomorphic to the category whose objects are the reduced $\mathbf{R}$-logics and whose arrows are all the logical morphisms between them.

(4) The category whose objects are the reduced $\mathbf{R}$-logics and whose arrows are the logical epimorphisms between them is a full reflective subcategory of the category whose objects are all $\mathbf{R}$-logics and whose arrows are the logical epimorphisms between them; the reflector is the functor associated with the process of reduction $\mathbb{L} \mapsto \mathbb{L}^{*}$. 
Proof: (1) comes from Corollary 3.10 of [16], which uses in an essential way the fact that $\mathbf{R}$ is algebraizable. (2) comes from Theorems 2.28 and 2.29 of the same paper, or, alternatively, from our Corollaries 4.15.1 and 5.3. together with the observation that pairs of associated R-logics and WR-logics have the same Tarski congruence. (3) comes from Theorem 2.33 of [16]. Finally (4) comes from Theorem 2.34 of the same paper.

Notice that in (4) we had to restrict the arrows to be epimorphisms; this restriction was not necessary in the case of WR (Corollary 4.15.3) because WR is strongly selfextensional, which is certainly not the case of $\mathbf{R}$.

As a last application of Theorem 5.7 we will give an intrinsic characterization of the abstract logics in $\mathbb{R}(\mathfrak{A})$ which makes no reference to the logics in $\mathbb{W} \mathbb{R}(\mathfrak{A})$, and which in some sense is analogous to Theorem 2 of [6]. This characterization uses a kind of generalized deduction theorem of the following form: Let $\mathbb{L}=\langle\mathfrak{A}, \mathrm{C}\rangle$ be an abstract logic, and let $T \subseteq A$; we say that $\mathbb{L}$ satisfies the deduction theorem parametrically restricted to $T$ (PRDT for $T$ ) when there is a ternary polynomial $p(x, y, z)$ such that for any $X \cup\{a, b\} \subseteq A, b \in \mathrm{C}(X, a) \Longleftrightarrow$ there is some $c \in T$ with $p^{\mathfrak{A}}(a, b, c)$ $\in \mathrm{C}(X)$. Then we first have:

Theorem 5.12 Every $\mathbf{R}$-logic satisfies the PRDT for $\mathrm{C}(\varnothing)$, with $p(x, y, z)=(x \wedge$ $z) \rightarrow y$.

Proof: If $\mathbb{L}=\langle\mathfrak{A}, \mathrm{C}\rangle \in \mathbb{R}(\mathfrak{A})$ and $b \in \mathrm{C}(X, a)=\mathrm{C}^{\prime}\left(X \cup E\left(\mathbb{L}^{\prime}\right), a\right)$ then, by Proposition 4.5.1 we know that there is some $x \in \mathrm{C}^{\prime}(X)$ and some $e \in E\left(\mathbb{L}^{\prime}\right)$ such that $b \in \mathrm{C}^{\prime}(x, e, a)$; actually $x=x_{1} \wedge \ldots \wedge x_{n}$ for some $x_{i} \in X$, and the same for $e$, but $E\left(\mathbb{L}^{\prime}\right)$ is a closed set of $\mathrm{C}^{\prime}$ and thus it is closed under Conjunction. Putting $c=(x \rightarrow x) \wedge(e \rightarrow e)$ then as a consequence of Proposition 4.8.11, $(a \wedge c) \rightarrow b \in$ $\mathrm{C}^{\prime}((a \wedge c) \rightarrow x,(a \wedge c) \rightarrow e,(a \wedge c) \rightarrow a)$. On the other hand, by the selection of $c, x \rightarrow x \in \mathrm{C}^{\prime}(a \wedge c)$ and then by Propositions 4.5.3 and 4.8.5, $(a \wedge c) \rightarrow x \in \mathrm{C}^{\prime}(x)$; by similar processes one can prove that $(a \wedge c) \rightarrow e \in \mathrm{C}^{\prime}(e)$, and then it follows that $(a \wedge c) \rightarrow b \in \mathrm{C}^{\prime}(x, e,(a \wedge c) \rightarrow a) \subseteq \mathrm{C}^{\prime}\left(x, E\left(\mathbb{L}^{\prime}\right)\right)=\mathrm{C}(x) \subseteq \mathrm{C}(X)$. Finally the converse implication contained in the PRDT is a direct consequence of Proposition 4.5.2.

Then it results that this Deduction Theorem characterizes the $\mathbf{R}$-logics among the finitary abstract logics whose closed sets are $\mathbf{R}$-filters (that is, among the finitary models of $\mathbf{R}$, see Proposition 4.8.110):

Theorem 5.13 Let $\mathbb{L}=\langle\mathfrak{A}, \mathrm{C}\rangle$ be an abstract logic of type $(2,2,1)$ with $\mathfrak{A}=$ $\langle A, \wedge, \rightarrow, \neg\rangle$. Then $\mathbb{L} \in \mathbb{R}(\mathfrak{A})$, that is, $\mathbb{L} \in \mathcal{F M o d}_{\mathbf{R}} \mathfrak{A}$, if and only if the following conditions hold:

(1) $\mathrm{C}$ is finitary;

(2) $C \subseteq \mathcal{F}_{\mathbf{R}} \mathfrak{A}$; and

(3) $\mathbb{L}$ satisfies the PRDT for $\mathrm{C}(\varnothing)$ with $p(x, y, z)=(x \wedge z) \rightarrow y$.

Proof: If $\mathbb{L}=\langle\mathfrak{A}, \mathrm{C}\rangle \in \mathbb{R}(\mathfrak{A})$, then Condition (1) is automatically satisfied, and

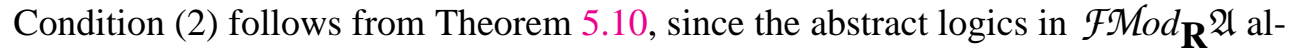
ways satisfy it; finally Condition (3) is proved in Theorem 5.12.

To prove the converse it will be enough to see that Conditions (1), (2) and (3) imply that $\mathfrak{A}^{*} \in \mathrm{R}$ and $C^{*}=\mathcal{D e d} \mathfrak{A}^{*}$, and then use Theorem 5.7. First of all ob- 
serve that by (2) $\langle\mathfrak{A}, \mathrm{C}(\varnothing)\rangle \in$ Matr $\mathbf{R}$ and that the algebraizability of $\mathbf{R}$ implies that the relation $\Omega_{\mathfrak{A}}(\mathrm{C}(\varnothing))$ is analytical, therefore by [7], Theorem IX.6, we have that $\Omega_{\mathfrak{A}}(\mathrm{C}(\varnothing))=\widetilde{\Omega}(\mathbb{L})$. Now let $\varphi \approx \psi$ be any equation valid in $\mathrm{R}$. Again by the algebraizability of $\mathbf{R}$ with respect to $\mathrm{R}$ with equivalence formula $p \leftrightarrow q$ (see Section 2 ) it follows that $\vdash_{\mathbf{R}} \varphi \leftrightarrow \psi$, therefore for any interpretation $\bar{a}$ over $\mathfrak{A}$ we have that $\varphi^{\mathfrak{A}}(\bar{a}) \leftrightarrow \psi^{\mathfrak{A}}(\bar{a}) \in \mathrm{C}(\varnothing)$, which is equivalent to $\left\langle\varphi^{\mathfrak{A}}(\bar{a}), \psi^{\mathfrak{A}}(\bar{a})\right\rangle \in \Omega_{\mathfrak{A}}(\mathrm{C}(\varnothing))$, that is, to $\left\langle\varphi^{\mathfrak{A}}(\bar{a}), \psi^{\mathfrak{A}}(\bar{a})\right\rangle \in \widetilde{\Omega}(\mathbb{L})$, and this means that $\varphi^{\mathfrak{A}^{*}}(\bar{a})=\psi^{\mathfrak{A}^{*}}(\bar{a})$. This proves that the equation $\varphi \approx \psi$ holds in $\mathfrak{A}^{*}$, which means that $\mathfrak{A}^{*} \in \mathrm{R}$. On the other hand, $C^{*} \subseteq \mathcal{F} i_{\mathbf{R}^{2}} \mathfrak{A}^{*}$ by (2), and by Theorem 2.4. $\mathcal{F} i \mathbf{R}^{\mathfrak{A}^{*}}=\operatorname{Ded}^{*}$; thus it only remains to prove that $\operatorname{Ded}^{*} \subseteq C^{*}$. Let $F \in \mathcal{D e d}^{*}$ and put $G=\pi^{-1}[F]$ where $\pi: \mathfrak{A} \rightarrow \mathfrak{A}^{*}$ is the canonical projection. We have $G / \widetilde{\Omega}(\mathbb{L})=F$, and it will be enough to prove that $G \in C$. Let $a \in \mathrm{C}(G)$; since (2) implies that $\mathrm{C}$ has the property of Conjunction DM2, then by (1) and (2) there are $a_{1}, \ldots, a_{n} \in G$ such that, putting $b=a_{1} \wedge \ldots \wedge a_{n}$, it holds that $a \in \mathrm{C}(b)$; but now by (3) there is some $c \in \mathrm{C}(\varnothing)$ such that $(b \wedge c) \rightarrow a \in \mathrm{C}(\varnothing)$; therefore, $(a \wedge(b \wedge c)) \leftrightarrow(b \wedge c) \in \mathrm{C}(\varnothing)$ and so $a^{*} \wedge\left(b^{*} \wedge c^{*}\right)=(a \wedge(b \wedge c)) / \widetilde{\Omega}(\mathbb{L})=(b \wedge c) / \widetilde{\Omega}(\mathbb{L})=b^{*} \wedge c^{*}$, that is, in $\mathfrak{A}^{*} \in \mathrm{R}$ we have $b^{*} \wedge c^{*} \leq a^{*}$. Since $F \in \mathcal{D e d}^{*}, b^{*} \in F$; now also $c^{*} \in F$. Using that $c \in \mathrm{C}(\varnothing)$ and miscellaneous properties of $\mathbf{R}$-filters one easily proves that $(c \rightarrow c) \leftrightarrow(c \wedge(c \rightarrow c)) \in \mathrm{C}(\varnothing)$; therefore $c^{*} \rightarrow c^{*} \leq c^{*}$ and $c^{*} \in E\left(\mathfrak{A}^{*}\right) \subseteq F$. As a consequence, $a^{*} \in F$, which implies $a \in G$. Thus we have proved that $\mathcal{D e d} \mathfrak{A}^{*}=C^{*}$, which finishes the proof.

This Parametrically Restricted Deduction Theorem can be related to the parameterized local deduction theorems studied in Czelakowski and Dziobiak [10], 11] (see also [9]); notice that $\mathbf{R}$ is one of the few examples of deductive systems which really need parameters in their deduction theorems. In turn, these results can be related to those in Meyer [22], where he studies the following implication:

$$
\varphi \rightarrow_{i} \psi=\varphi \wedge \mathbf{t} \rightarrow \psi
$$

in the conservative extension $\mathbf{R}_{\mathbf{t}}$ of $\mathbf{R}$ (see Section 2); in his Lemma 2 he proves that this connective satisfies the ordinary Deduction Theorem. Taking out the constant $\mathbf{t}$ leads us to a parametric restriction, either in the form of Czelakowski or in the form we have used above.

Acknowledgments Financial help for the writing of this paper was obtained from grant PB 90-0465-C02-01 of the Spanish DGICYT.

\section{REFERENCES}

[1] Anderson, A. R. and N. D. Belnap, Entailment: The Logic of Relevance and Necessity, vol. 1, Princeton University Press, 1975. Z Zbl 0323.02030 MR 53:10542 1, 1, 2, 2, 2, $2,2,2,2,2$

[2] Anderson, A. R., N. D. Belnap, and J. M. Dunn, Entailment: The Logic of Relevance and Necessity, vol. 2, Princeton University Press, 1992.Zbl 0921.03025|MR 94b:03042 1.11.1.2 
[3] Balbes, R. and P. Dwinger, Distributive lattices, University of Missouri Press, Columbia, 1974. Zbl 0321.06012||MR 51:10185 1,2

[4] Blok, W. J. and D. Pigozzi, "Protoalgebraic logics," Studia Logica, vol. 45 (1986), pp. 337-369. Zbl 0622.03020 MR 88e:03045 1.3

[5] Blok, W. J. and D. Pigozzi, Algebraizable logics, Memoirs of the American Mathematical Society, vol. 77, no. 396, American Mathematical Society, Providence, 1989. Zbl 0664.03042|MR 90d:03140 1.1.11.1.12.2.2.2.3.3

[6] Bloom, S. L. and D. J. Brown, "Classical Abstract Logics," Dissertationes Mathematicae, vol. 102 (1973), pp. 43-51.Zbl 0317.02072 1,5,5,5,5

[7] Brown, D. J. and R. Suszko, “Abstract Logics,” Dissertationes Mathematicae, vol. 102 (1973), pp. 9-42.Zbl 0317.02071||MR 56:5284 1,1,4,4,5,5

[8] Burris, S. and H. P. Sankappanavar, A Course in Universal Algebra, Springer-Verlag, New York, 1981. Zbl 0478.08001 MR 83k:08001 1

[9] Czelakowski, J., Consequence Operations. Foundational Studies, Prepublication, Polish Academy of Sciences, Warszawa, 1992. 1.3.5

[10] Czelakowski, J. and W. Dziobiak, "The deduction-like theorem for quasivarieties of algebras and its application," pp. 8-14 in Algebraic Logic, edited by H. Andréka, J .D. Monk, and I. Németi, Colloquia Mathematica Societatis János Bolyai, vol. 54, North-Holland, Amsterdam, 1991. 3,5

[11] Czelakowski, J. and W. Dziobiak, “A deduction theorem schema for deductive systems of propositional logic," Studia Logica, vol. 50 (1991), pp. 385-390. Zbl 0755.03014 MR 93e:03012 5

[12] Došen, K., "Modal translations in substructural logics," Journal of Philosophical Logic, vol. 21 (1992), pp. 283-336.Zbl 0765.03009 MR 93i:03033 4

[13] Došen, K. and P. Schroeder-Heister, editors, Substructural Logics, Clarendon Press, Oxford, 1993. Zbl 0811.68056|MR 95a:03003 1

[14] Dunn, J. M., The algebra of intensional logics, Ph. D. Dissertation, University of Pittsburgh, 1966.

[15] Font, J. M., “On the Leibniz congruences," pp. 17-36 in Algebraic Methods in Logic and Computer Science, edited by C. Rauszer, (Banach Center Publications, vol. 28) Polish Academy of Sciences, Warszawa, 1993.Zbl 0796.03008|MR 97m:03021]1,4,4

[16] Font, J. M. and R. Jansana, "A General Algebraic Semantics for Deductive Systems," Mathematics Preprint Series, vol. 126, University of Barcelona, February 1993. 1.1. $4,4,4,4,4,4,4,4,4,4,4,5,5,5,5,15,15,5,15,5$

[17] Font, J. M. and G. Rodríguez, "Note on algebraic models for relevance logic," Zeitschrift für Mathematische Logik und Grundlagen der Mathematik, vol. 36 (1990),

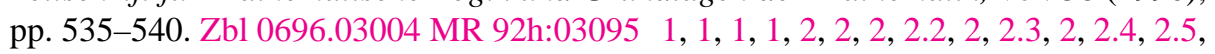
$2,3,3,4$

[18] Font, J. M. and V. Verdú, “Abstract characterization of a four-valued logic,” pp. 389396 in Proceedings of the 18th International Symposium on Multiple-Valued Logic, IEEE Computer Society, Palma de Mallorca, 1988. 1.4.14.14.4.14.14.14

[19] Font, J. M. and V. Verdú, “Completeness theorems for a four-valued logic related to De Morgan lattices,” Mathematics Preprint Series, vol. 57, University of Barcelona, March 1989. 4,4 
[20] Font, J. M. and V. Verdú, "A first approach to abstract modal logics," Journal of Symbolic Logic, vol. 54 (1989), pp. 1042-1062.ZZbl 0687.03008 MR 90j:03031

[21] Font, J. M. and V. Verdú, "Algebraic logic for classical conjunction and disjunction," Studia Logica, vol. 50 (1991), pp. 391-419. Zbl 0753.03027|MR 94a:03007 1

[22] Meyer, R. "Intuitionism, entailment, negation," pp. 168-198 in Truth, Syntax and Modality, edited by H. Leblanc, North-Holland, Amsterdam, 1973.Zbl 0317.02016 MR 53:95 5

[23] Ono, H., "Semantics for substructural logics," pp. 259-291 in Substructural Logics, edited by K. Došen and P. Schroeder-Heister, Clarendon Press, Oxford, 1993. Zbl 0941.03522||MR 95f:03013,4

[24] Slaney, J. K., "Reduced models for relevant logics without WI," Notre Dame Journal of Formal Logic, vol. 28 (1987), pp. 395-407.

[25] Smiley, T., "The independence of connectives," The Journal of Symbolic Logic, vol. 27 (1962), pp. 426-436. Zbl 0139.00601|lMR 30:3003 1

[26] Wójcicki, R., Lectures on Propositional Calculi, Ossolineum, Wroclaw, 1984. Zbl 0647.03019||MR 86h:03002 1.1.11

[27] Wójcicki, R., Theory of Logical Calculi: Basic Theory of Consequence Operations, (Synthese Library, vol. 199) Reidel, Dordrecht, 1988. 1.11.1.,3.]3.|3.14

Departament de Lògica, Història i Filosofia de la Ciència

Facultat de Matemàtiques

Universitat de Barcelona

Gran Via 585, 08007 Barcelona, Spain

email: font @ cerber.mat.ub.es

Seminari de Matemàtiques

E. U. Estudis Empresarials

Universitat de Barcelona

Diagonal 696, 08034 Barcelona, Spain 